\title{
Paires de structures de contact sur les variétés de dimension trois
}

\author{
VINCENT COLIN \\ SEBASTIÃO FIRMO
}

\begin{abstract}
On introduit une notion de paire positive de structures de contact sur les variétés de dimension trois qui généralise celle de Eliashberg and Thurston [5] and Mitsumatsu $[13 ; 14]$. Une telle paire "normale" donne naissance à un champ de plans continu et localement intégrable $\lambda$. On montre que si $\lambda$ est uniquement intégrable et si les structures de contact sont tendues, alors le feuilletage intégral de $\lambda$ est sans composante de Reeb d'âme homologue à zéro. De plus, dans ce cas, la variété ambiante porte un feuilletage sans composante de Reeb. On démontre également un théorème de stabilité "à la Reeb" pour les paires positives de structures tendues.

We introduce the notion of a positive pair of contact structures of a three dimensional manifold which generalises that of $[5 ; 13 ; 14]$. A normal such pair gives rise to a continuous, locally integrable plane field $\lambda$. We show that if $\lambda$ is uniquely integrable and if the contact structures are tight then the integral foliation of $\lambda$ has no Reeb component whose core is homologous to zero. Moreover, in this case, the ambient manifold carries a foliation without a Reeb component. We also show a Reeb stability theorem for positive pairs of tight structures.
\end{abstract}

57M50, 57R17, 57R30

\section{Introduction}

En dimension trois, Eliashberg et Thurston [5] et Mitsumatsu [13; 14] associent à toute paire $\left(\xi_{+}, \xi_{-}\right)$de structures de contact transversales, où $\xi_{+}$est positive et $\xi_{-}$ négative, une paire de champs de plans $\left(\lambda_{+}, \lambda_{-}\right)$transversaux, continus et localement (non uniquement) intégrables: par tout point passe un germe de surface intégrale de $\lambda_{ \pm}$. L'intersection $\xi_{+} \cap \xi_{-}$est alors dirigée par un champ de vecteurs "conformément" Anosov. Réciproquement, cette situation se rencontre lorsqu'on étudie les directions stables et instables $\lambda_{ \pm}$d'un champ de vecteurs Anosov, les structures $\xi_{ \pm}$apparaissant comme "plans médians" des $\lambda_{ \pm}$.

Du point de vue de la géométrie de contact, le fait d'imposer à $\xi_{+}$et $\xi_{-}$d'être des structures transversales est trop contraignant. Le but du présent article est d'étudier le cas de paires $\left(\xi_{+}, \xi_{-}\right)$où l'on relâche cette condition pour la remplacer par une propriété de 
coïncidence positive: les champs de plans $\xi_{+}$et $\xi_{-}$ne sont jamais "dos-à-dos". Autrement dit, ils sont transversaux à un même champ de droites $\mathcal{D}$. Dans cette situation, on perd l'existence d'un des deux champs intégrable $\lambda_{ \pm}$, mais on en préserve un, $\lambda$, coincé entre $\xi_{+}$et $\xi_{-}$et lui aussi transversal à $\mathcal{D}$. Comme auparavant, le champ $\lambda$ est localement intégrable et en général seulement continu: il n'y a pas unicité locale des surfaces intégrales, ni donc a priori de feuilletage intégral global. Lorsque cette unicité est avérée, par exemple sous les conditions du théorème 3.4, les propriétés de rigidité des structures $\xi_{+}$et $\xi_{-}$rejaillissent sur celle du feuilletage intégral: si celles-là sont tendues, celui-ci est sans composante de Reeb d'âme homologue à zéro et la variété ambiante porte un feuilletage sans composante de Reeb (théorème 3.3). Notre leitmotiv est que cette notion de paire positive de structures de contact tendues pourrait être une bonne généralisation de celle de feuilletage sans composante de Reeb. En particulier, d'après Eliashberg et Thurston [5], tout feuilletage tendu (et même tout feuilletage sans composante de Reeb; voir Colin [2]) est limite de paires positives de structures de contact tendues. Pour conclure, on démontre pour les paires positives de structures de contact tendues un analogue du théorème de stabilité de Reeb pour les feuilletages (théorème 4.1). Ce résultat semble faire écho à la théorie des courbes holomorphes. Son pendant a en effet été démontré par Eliashberg et Hofer [4], via une méthode de remplissage par des disques holomorphes. Par certains aspects, les résultats de cet article peuvent être vus comme une version topologique des feuilletages d'énergie finie de Hofer, Wyzocki et Zehnder [12]. La notion de paire positive est également bien adaptée à celle de surface branchée, connue dans le monde intégrable pour rendre compte des propriétés des laminations. Zannad tire bénéfice de ce parallèle dans [17; 18] en trouvant une condition suffisante, inspirée de la géométrie de contact, pour qu'une surface branchée porte une lamination.

Remerciements Ce travail a été rendu possible par l'accord France-Brésil, grâce auquel nous avons pu séjourner à l'Université Federal Fluminense et à l'Université de Nantes. Il a également bénéficié du support de l'Institut Universitaire de France et de l'ANR Symplexe. Nous souhaitons saluer ces institutions pour leur soutien. Le rapporteur anonyme d'une première version de ce texte y a relevé de nombreuses erreurs. Cette nouvelle mouture doit beaucoup à la qualité de son travail. Nous lui adressons nos plus vifs remerciements.

\section{Paires de structures de contact}

\subsection{Structures de contact}

Une structure de contact (orientable) sur une variété orientée $V$ de dimension trois est un champ de plans orientable lisse $\xi$, noyau d'une 1 -forme $\alpha$ dont le produit 
extérieur avec $d \alpha$ ne s'annule pas. Le signe de $\xi$ est celui de $\alpha \wedge d \alpha$, rapporté à l'orientation de $V$. À l'opposé des feuilletages, les structures de contact supportent bien les déformations: la condition de contact est ouverte pour la topologie $C^{1}$ et, d'après un théorème de Gray [9], tout chemin de structures de contact est le fait d'une isotopie de $V$ issue de l'identité.

Une structure de contact $\xi$ est tendue si aucun disque plongé dans $V$ ne s'appuie sur une courbe intégrale de $\xi$ transversalement à $\xi$. Dans le cas contraire, on dit que $\xi$ est vrillée. Une structure de contact est universellement tendue lorsque son rappel dans le revêtement universel de $V$ est tendu.

Soit $\gamma$ une courbe plongée dans $V$ qui borde une surface compacte, plongée et orientable $S$. Si $\gamma$ est transversale à $\xi$, l'autoenlacement $l(\gamma)$ de $\gamma$ est l'enlacement entre $\gamma$ et toute courbe obtenue en poussant légèrement $\gamma$ par une section non singulière de $\left.\xi\right|_{S}$. De même, si $\gamma$ est legendrienne, c'est-à-dire tangente à $\xi$, son invariant de Thurston-Bennequin est l'entier $\operatorname{tb}(\gamma)$ obtenu en comptant l'enlacement entre $\gamma$ et sa déformation dans la direction d'un vecteur normal à $\xi$. Ces enlacements sont calculés avec l'orientation de $V$ qui rend $\xi$ positive. Lorsque $\xi$ est tendue, si $\chi(S)$ désigne la caractéristique de $S$, l'autoenlacement $l(\gamma)$ et l'invariant de Thurston-Bennequin $\operatorname{tb}(\gamma)$ vérifient les inégalités de Bennequin [1]:

$$
l(\gamma) \leq-\chi(S) \quad \text { et } \quad \operatorname{tb}(\gamma) \leq-\chi(S)
$$

Si $S$ est une surface close orientée, munie d'une forme d'aire $\omega$, et plongée dans une variété de contact $(V, \xi)$, dont l'orientation est donnée par $\xi$, on appelle feuilletage caractéristique de $S$, noté $\xi S$, le feuilletage intégral du champ de vecteurs $Y$ donné par

$$
i_{Y} \omega=\left.\alpha\right|_{T S}
$$

qui dirige $\xi \cap T S$. Ses singularités sont les points $x \in S$ où $\xi(x)=T_{x} S$.

Remarque 1 On insiste sur le fait que pour déterminer l'orientation du feuilletage caractéristique d'une surface $S$, on utilise une orientation de l'espace ambiant différente suivant que la structure de contact $\xi$ considérée est positive ou négative. La divergence d'une singularité du feuilletage caractéristique sera donc toujours positive là où les orientations de la structure $\xi$ et de $T S$ coïncident, ce indépendemment du signe de $\xi$.

\subsection{Paires de structures}

Soit $V$ une variété de dimension trois orientée. On appelle paire de structures de contact la donnée d'une structure $\xi_{+}$positive et d'une structure $\xi_{-}$négative. 
Dans la suite, on ne considère que des structures $\xi_{+}$et $\xi_{-}$coorientées (et donc orientées). On appelle point de contact entre $\xi_{+}$et $\xi_{-}$un point $x \in V$ où $\xi_{+}(x)=\xi_{-}(x)$. Les points de contact sont de deux sortes: positifs si les coorientations de $\xi_{+}$et $\xi_{-}$coïncident en $x$, et négatifs si elles sont opposées. Pour une paire $\left(\xi_{+}, \xi_{-}\right)$, on note $\Delta_{+}$le lieu des contacts positifs, $\Delta_{-}$le lieu des contacts négatifs et $\Delta=\Delta_{+} \cup \Delta_{-}$.

Soient $\alpha_{+}$et $\alpha_{-}$des équations de $\xi_{+}$et $\xi_{-}$, toujours supposées positives sur un vecteur normal direct a, respectivement, $\xi_{+}$et $\xi_{-}$. Il existe un unique champ de vecteurs $X$ inclus dans $\xi_{-}$et qui vérifie l'équation

$$
\left.i_{X} d \alpha_{-}\right|_{\xi_{-}}=\left.\alpha_{+}\right|_{\xi_{-}} .
$$

Il est lisse, nul le long de $\Delta$ et dirige $\xi_{+} \cap \xi_{-}$sur $V \backslash \Delta$. Sa divergence dans la direction de $\xi_{-}$pour la forme $d \alpha_{-}$est bien définie et non nulle aux points où $\xi_{+}=\xi_{-}$: $\mathcal{L}_{X} d \alpha_{-}\left|\xi_{-}=d\left(i_{X} d \alpha_{-}\right)\right|_{\xi_{-}}=\left.d \alpha_{+}\right|_{\xi_{+}}$. En un point de $\Delta_{+}$(resp. $\Delta_{-}$), la divergence de $X$ dans la direction de $\xi_{ \pm}$est positive (resp. négative).

Génériquement $\Delta$ est un entrelacs. Dans cette section, on montre que

- par isotopie de $\xi_{+}$, on peut rendre la paire $\left(\xi_{+}, \xi_{-}\right)$normale (proposition 2.1), c'est-à-dire obtenir un contrôle du champ de vecteurs $X$ près de $\Delta$ (en faisant ressembler le feuilletage dirigé par $X$ à la famille à 1 -paramètre de feuilletages

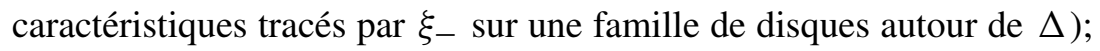

- une paire normale donne naissance à des champs de plans $\lambda_{ \pm}^{ \pm \infty}$ (proposition 2.4) qui sont continus et localement intégrables (proposition 2.5).

\subsection{Paires normales}

On note $D^{2}=\left\{(x, y) \in \mathbb{R}^{2}, x^{2}+y^{2} \leq 1\right\}$ le disque unité de $\mathbb{R}^{2}$.

Une paire $\left(\xi_{+}, \xi_{-}\right)$, définie sur une variété close (compacte sans bord) $V$, sera dite normale si l'ensemble $\Delta$ est un entrelacs lisse plongé dans $V$, transversal à $\xi_{ \pm}$sauf en un nombre fini de points $\left(x_{i}\right)_{1 \leq i \leq n}$, et qui vérifie les propriétés suivantes.

- Si $a_{i}$ désigne un arc ouvert transversal à $\xi_{ \pm}$et délimité par $x_{i}$ et $x_{i+1}$ dans $\Delta$, alors $a_{i}$ possède un voisinage tubulaire $D^{2} \times a_{i},\{(0,0)\} \times a_{i}=a_{i}$, sur lequel $\xi_{+} \cap \xi_{-} \subset T D^{2} \times\{\mathrm{pt}\}$ et le feuilletage caractéristique $\xi_{+} D^{2} \times\{\mathrm{pt}\}$ est égal au feuilletage $\xi_{-} D^{2} \times\{\mathrm{pt}\}$ (égalité dans $\Delta_{+}$, égalité à orientation près dans $\Delta_{-}$) et est un feuilletage de $D^{2} \times\{\mathrm{pt}\}$ par des selles ou par des foyers radiaux.

- La sous-variété $\Delta$ a un contact quadratique avec $\xi_{ \pm}$en les $x_{i}$, et $x_{i}$ possède un voisinage $D^{2} \times[-1,1], x_{i}=(0,0,0)$, où $\xi_{+} \cap \xi_{-}=\xi_{+} \cap T D^{2} \times\{t\}=\xi_{-} \cap T D^{2} \times\{t\}$, et où le feuilletage caractéristique de $\xi_{+} D^{2} \times\{t\}=\xi_{-} D^{2} \times\{t\}$, pour $t \in[-1,1]$, est 
le film d'une élimination entre un foyer et une selle. En particulier, le feuilletage de $D^{2} \times\{0\}$ possède une singularité de type naissance-mort en $(0,0,0)=x_{i}$. Dans ces coordonnées, le champ de vecteurs $X$ est tangent au champ de droites $\{d t=d y=0\}$ sur $\{y=0\} \times[-1,1]$ (l'élimination se fait le long de ces caractéristiques).

On montre que toute paire se laisse déformer en une paire normale.

Proposition 2.1 Sur une variété close $V$, pour toute paire $\left(\xi_{+}, \xi_{-}\right)$il existe une paire normale $\left(\xi_{+}^{\prime}, \xi_{-}^{\prime}\right)$ où $\xi_{ \pm}^{\prime}$ est isotope à $\xi_{ \pm}$.

Démonstration On fixe une trivialisation de $T V$, ainsi qu'une métrique sur $V$. La donnée de $\xi_{+}$et $\xi_{-}$est la donnée de deux applications $f_{ \pm}: V \rightarrow S^{2} \subset \mathbb{R}^{3}$. La condition de contact est ouverte pour la topologie $C^{1}$, et même, d'après le théorème de Gray, deux applications $f$ et $g$ qui sont $C^{1}$-proches donnent des structures de contact isotopes. On peut donc, quitte à perturber $\xi_{+}$par isotopie, se placer dans la situation générique (*) où l'application $F: V \rightarrow S^{2} \times S^{2}, F(x)=\left(f_{+}(x), f_{-}(x)\right)$, est transversale à la diagonale et à l'antidiagonale de $S^{2} \times S^{2}$. Sous cette hypothèse, $\Delta_{+}$ et $\Delta_{-}$sont des sous-variétés de dimension 1 de $V$, et le long de $\Delta$ la différentielle de $X$ est de rang 2. On suppose également vérifiée la propriété générique: les points où $\Delta_{+}$et $\Delta_{-}$sont tangents à $\xi_{ \pm}$sont des contacts quadratiques et donc isolés. Les composantes de $\Delta \backslash\left(\bigcup_{1 \leq i \leq n} x_{i}\right)$ sont de deux types suivant le signe du déterminant de la différentielle de $X$ dans la direction normale: branches de foyers si ce déterminant est positif et branches de selles s'il est négatif.

On suppose que $\Delta_{+}$a des contacts quadratiques avec $\xi_{ \pm}$; dans le cas contraire, l'étude se simplifie. Un voisinage $N\left(\Delta_{+}\right)$de $\Delta_{+}$est dit normal s'il est constitué des éléments suivants:

- un voisinage $N\left(x_{i}\right)$ de chaque point de contact quadratique $x_{i}, i=1, \ldots, n$, de la forme $N\left(x_{i}\right)=\left\{|x| \leq z_{0},|y| \leq \sqrt{z_{0}}+z_{0},|z| \leq z_{0}\right\}$, où $z_{0}>0$ et $\xi_{ \pm}\left(x_{i}\right)=\operatorname{ker} d z$. Le champ de vecteurs $X$ est transversal aux faces verticales $\left\{|x|=z_{0}\right\}$ et $\left\{|y|=\sqrt{z_{0}}+z_{0}\right\}$. Il est horizontal (ie inclus dans ker $d z$ ) et sort de $N\left(x_{i}\right)$ le long de ces faces verticales, sauf le long de la face $\left\{y=\sqrt{z_{0}}+z_{0}\right\}$ où il rentre dans $N\left(x_{i}\right)$. Pour tout $c \in\left[-z_{0}, z_{0}\right]$, l'arc $\left\{|x|=z_{0}, z=c\right\} \cup\left\{y=-\sqrt{z_{0}}-z_{0}, z=c\right\}$ (orienté comme bord du disque horizontal, lui-même coorienté par $\partial_{z},\{z=c\}$ ) est positivement transversal à $\xi_{+}$ et, sauf aux deux points $\left\{x= \pm z_{0}, y=0, z=-z_{0}\right\}$, son intérieur est négativement transversal à $\xi_{-}$. L'arc $\left\{y=\sqrt{z_{0}}+z_{0}, z=c\right\}$ est négativement transversal à $\xi_{+}$et tangent à $\xi_{-}$. En particulier, du fait que $X$ y est horizontal, $\xi_{-}$a pour équation $d z=0$ le long de cet arc. La structure $\xi$ - est également d'équation $d z=0$ le long de l'arc $\left\{y=0, z=-z_{0}\right\}$, tandis que $\xi_{+}$lui est transversal. Pour finir, le champ $X$ dirige le feuilletage caractéristique de la face supérieure $\left\{z=z_{0}\right\}$ pour $\xi_{+}$et $\xi_{-}$(seulement en 
dehors de l'arc $\left\{y=\sqrt{z_{0}}+z_{0}\right\}$ pour cette dernière, qui est, d'après ce qui précède, une ligne de singularités de $\xi-\left\{z=z_{0}\right\}$ ).

- un voisinage tubulaire $N=\bigcup_{1 \leq j \leq n} N_{j}$ de la collection $\Delta_{+} \backslash \bigcup_{1 \leq i \leq n} \operatorname{Int}\left(N\left(x_{i}\right)\right)$ d'arcs, où les voisinages $N_{j} \simeq[-1,1] \times[-1,1] \times[0, m]$ sont deux à deux disjoints. Chaque $N_{j}$ est muni de coordonnées $(x, y, z)$ avec $\Delta_{+} \cap N_{j}=(0,0) \times[0, m]$. Il intersecte $N\left(x_{i}\right), i=1, \ldots, n$, le long du disque $\left\{-z_{0}-\sqrt{z_{0}} \leq y \leq 0, z=-z_{0}\right\} \subset$ $N\left(x_{i}\right)$, du disque $\left\{0 \leq y \leq \sqrt{z_{0}}+z_{0}\right\} \subset N\left(x_{i}\right)$, ou de l'ensemble vide. S'il s'agit du voisinage d'une branche de foyers, il est feuilleté par des disques horizontaux $\{z=c\}$ dont les bords sont positivement transversaux à $\xi_{+}$et négativement transversaux à $\xi_{-}$ (le champ de vecteurs $X$ est horizontal et sortant le long du bord), sauf en $\{z=0\}$ et $\{z=m\}$ où une arête est tangente à $\xi_{-}$. S'il s'agit du voisinage d'une branche de selles, le champ $X$ est horizontal et rentrant le long des faces verticales $\{|y|=1\}$, et horizontal et sortant le long des faces verticales $\{|x|=1\}$. La structure $\xi_{+}$est

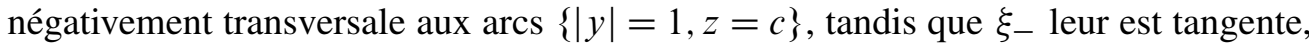
et positivement transversale aux $\operatorname{arcs}\{|x|=1, z=c\}$, tandis que $\xi$ - est négativement transversale à leur intérieur.

En renversant le sens de $X$, on définit de façon similaire une notion de voisinage normal pour $\Delta_{-}$.

Lemme 2.2 Si $\left(\xi_{+}, \xi_{-}\right)$est une paire de structures de contact générique pour laquelle $\Delta$ est une sous-variété de dimension 1 qui a des contacts quadratiques avec $\xi_{ \pm}$, alors $\Delta$ possède un voisinage normal.

Démonstration On explique comment trouver un voisinage normal de $\Delta_{+}$. L'étude pour $\Delta_{-}$est similaire.

Soit $p$ un point de contact quadratique entre $\Delta_{+}$et $\xi_{ \pm}$. On prend un voisinage $U_{0} \subset \mathbb{R}^{3}$ de $p=(0,0,0)$, muni de ses coordonnées cartésiennes $(x, y, z)$, pour lequel $\xi_{ \pm}(p)=\{d z=0\}$. Par commodité, on se référera à la coordonnée $z$ comme à la direction verticale, et aux coordonnées $x$ et $y$ comme aux directions horizontales. Soit $X$ le champ de vecteurs lisse, qui dirige $\xi_{+} \cap \xi_{-}$en dehors de $\Delta$ et nul sur $\Delta$, défini par l'équation $\left.i_{X} d \alpha_{-}\right|_{\xi_{-}}=\left.\alpha_{+}\right|_{\xi_{-}}$. On écrit le développement de $X$ à l'ordre 1 au voisinage de $(0,0,0): X=X_{1}+o(\|(x, y, z)\|)$. Comme $X$ est dans $\xi_{+}$, il vérifie une équation $d z=f d x+g d y$, avec $f(0,0, z)=g(0,0, z)=0$. En particulier, $X_{1}$ est horizontal: $X_{1}=\left(a_{0} x+b_{0} y+c_{0} z\right) \partial_{x}+\left(a_{1} x+b_{1} y+c_{1} z\right) \partial_{y}$. Comme le rang de $D X_{1}$ en $p$ est 2 , on obtient, après un changement de variables dans $T_{p} V$ qui donne une forme réduite de Jordan à $D_{p} X$, que $X_{1}$ est du type $X_{1}=a x \partial_{x}+b z \partial_{y}$. Une fois choisi $\left(\partial_{x}, \partial_{y}, \partial_{z}\right)$ dans $T_{p} V$, on étend ce repère par de nouvelles coordonnées $(x, y, z)$ de sorte que $\Delta_{+}=\left\{z=-y^{2}, x=0\right\}$ et $\xi_{-}=d z-x d y$ près de $p$. 
Le développement de $X$ à un ordre supérieur est, dans ces coordonnées:

$$
\begin{array}{r}
X=\left(x\left(R_{1}(x, y, z)+\left(z+y^{2}\right) R_{2}(x, y, z)\right) \partial_{x}+\left(\left(z+y^{2}\right) R_{3}(x, y, z)+x R_{4}(x, y, z)\right) \partial_{y}\right. \\
+\left(b x\left(z+y^{2}\right)+x^{2} R_{4}(x, y, z)\right) \partial_{z}+o\left(\|(x, y, z)\|^{2}\right)
\end{array}
$$

où

- $R_{1}, R_{2}, R_{3}$ et $R_{4}$ sont d'ordre 1 ;

- $R_{1}$ et $R_{3}$ ont un terme constant non nul (resp. $a$ et $b$ ), à l'inverse de $R_{2}$ et $R_{4}$;

- les termes d'ordre 3 provenant de $y^{2} R_{2}$ et $y^{2} R_{3}$ sont ceux apparaissant dans le développement de $X$ à l'ordre 3 .

Pour fixer les idées, on se place dans le cas où $b>0$, ce qui signifie que $\Delta_{+}$se situe dans la zone $z \leq 0$.

On considère alors le voisinage $U$ de $p$ donné par $\left\{|x| \leq z_{0},|y| \leq \sqrt{z_{0}}+z_{0},|z| \leq z_{0}\right\}$, où $z_{0}>0$ est choisi assez petit.

Dans l'écriture de la coordonnée de $X$ sur $\partial_{x}$, les termes en $z^{2}, y z$ et $y^{3}$ sont toujours au maximum de l'ordre de $\left(z_{0}\right)^{1 / 2+1}$, c'est-à-dire négligeables devant $z_{0}$. De même, lorsque $|y|=\sqrt{z_{0}}+z_{0}, z+y^{2}$ est toujours supérieur à un $O\left(z_{0}\right)$; c'est donc le terme dominant dans le coefficient de $\partial_{y}$. On vérifie ainsi que pour $z_{0}$ assez petit, $X$ est transversal aux faces verticales $|x|=z_{0}$ et $|y|=\sqrt{z_{0}}+z_{0}$. Il sort de $U$ le long de $|x|=z_{0}$ car $a>0$ (c'est la divergence de $X$ dans $\xi_{-}(p)$ ), sort de $U$ le long de $y=-\left(\sqrt{z_{0}}+z_{0}\right)$ et rentre dans $U$ le long de $y=\sqrt{z_{0}}+z_{0}$.

Lorsque le voisinage $U$ est assez petit ( $z_{0}$ est assez petit), $R_{3}(x, y, z)$ vaut environ $b$. De plus, comme la composante sur $\partial_{z}$ est environ $b x\left(z+y^{2}\right)$, toute orbite partant dans $U$ de l'altitude $z_{0}$ rencontre une des faces verticales (ou plutôt leur prolongement dans la direction des $z$ ) à une altitude supérieure à $z_{0}-O\left(z_{0} \sqrt{z_{0}}\right)$. Pour $z_{0}$ assez petit, une telle orbite sort à une altitude positive proche de $z_{0}$. On prend dans la face $\left\{y=\sqrt{z_{0}}+z_{0}\right\}$ l' $\operatorname{arc} A=\left\{z=\frac{3}{4} z_{0}\right\}$. Il est transversal (positivement) à $\xi_{+}$et tangent à $\xi_{-}$. On considère la surface constituée de la réunion des orbites de $X$ passant par $A$. De son intersection avec $U$, on ne garde que la composante qui contient $A$. La discussion effectuée plus haut assure que celle-ci est compacte, a son bord dans les faces verticales, est parallèle à la face $\left\{z=z_{0}\right\}$ et est contenue dans la région $z>0$. De plus, son feuilletage caractéristique pour $\xi_{+}$et $\xi_{-}$est dirigé par $X$ (en dehors de l'arc $A$ ). Elle découpe $U$ en deux composantes. On nomme $U^{\prime}$ celle qui contient $p$. Remplacer $U$ par $U^{\prime}$ revient à remplacer la face horizontale $\left\{z=z_{0}\right\}$ par une face

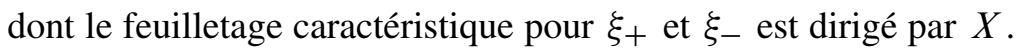

On change la structure produit de $U^{\prime}$ pour obtenir les propriétés de tangence et de transversalité des cercles horizontaux $\partial U^{\prime} \cap\{z=c\}$ par rapport à $\xi_{+}$et $\xi_{-}$. Pour cela, 
on utilise le fait que l'holonomie de $\xi_{+}$autour du bord vertical de $U^{\prime}$ est positive, tandis qu'elle est négative pour $\xi_{-}$. Ici l'holonomie de $\xi_{ \pm}$est définie comme l'application de premier retour du feuilletage caractéristique $\xi_{ \pm} \partial U^{\prime}$ sur une arête verticale, par exemple $\left\{x=-z_{0}, y=\sqrt{z_{0}}+z_{0}\right\}$. Elle est positive (resp. négative ou nulle) quand l'orbite issue d'un point $q$ revient à une altitude $z$ supérieure (resp. inférieure ou égale) à celle de $q$.

Si on suit le feuilletage caractéristique de $\xi$ - le long de la face verticale $\left\{y=z_{0}+\sqrt{z_{0}}\right\}$, puis le feuilletage de $\xi_{+}$le long des autres faces verticales, on obtient une holonomie encore supérieure à celle obtenue en suivant toujours le feuilletage caractéristique de $\xi_{+}$(car $X$ rentre dans $U^{\prime}$ le long de $\left\{y=z_{0}+\sqrt{z_{0}}\right\}$ et sort de $U^{\prime}$ le long des autres faces verticales). À l'inverse, si on suit le feuilletage de $\xi_{-}$, on obtient une holonomie négative. Par le théorème des valeurs intermédiaires, on est assuré d'obtenir une holonomie nulle, c'est-à-dire un feuilletage par cercles, en suivant le feuilletage de $\xi_{-}$sur la face $\left\{y=z_{0}+\sqrt{z_{0}}\right\}$ et en suivant une certaine trajectoire restant dans le cône délimité par $\xi_{+}$et $\xi_{-}$le long des autres faces verticales. On change les coordonnées pour que ces cercles deviennent les bords des disques horizontaux. En particulier, on change ainsi la face horizontale inférieure de $U^{\prime}$, en la faisant s'appuyer sur un des cercles décrit plus haut. L'argument développé ci-dessus permet également

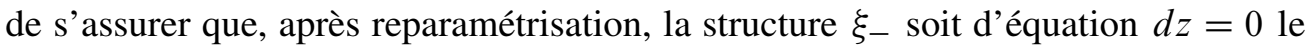
long de l'arc $\left\{y=0, z=-z_{0}\right\}$. Le voisinage obtenu est $N\left(x_{i}\right)$.

Soit maintenant $p$ un point de $\Delta_{+}$où $\Delta_{+}$est transversal à $\xi_{ \pm}$. Un voisinage de $p$ s'envoie sur $\mathbb{R}^{3}$ muni de coordonnées cartésiennes $(x, y, z)$ dans lesquelles

$$
p=(0,0,0), \quad \Delta_{+}=\{x=y=0\} \quad \text { et } \quad \xi_{ \pm}(0,0,0)=\{d z=0\} .
$$

On écrit à nouveau le développement de $X$ à l'ordre 1 au voisinage de $(0,0,0)$. Cette fois, on obtient que $X_{1}=\left(a_{0} x+b_{0} y\right) \partial_{x}+\left(a_{1} x+b_{1} y\right) \partial_{y}$. On est toujours dans la situation générique où la différentielle de $X$, et donc de $X_{1}$, est de rang 2 en $p$, c'est-à-dire que $a_{0} b_{1}-a_{1} b_{0} \neq 0$. Suivant le signe de ce déterminant, on est en présence d'une branche de foyers ou de selles. On trouve facilement un voisinage $N$ de cette branche avec les propriétés recherchées (voir la discussion du cas des voisinages $N\left(x_{i}\right)$

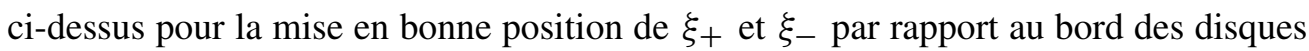
horizontaux).

Enfin, on peut vérifier qu'il est possible d'ajuster les choix de $U^{\prime}=N\left(x_{i}\right)$ et de $N$ afin qu'ils s'intersectent comme dans la définition de voisinage normal. La raison en est à nouveau que dans les voisinages $U$, la coordonnée de $X$ sur $\partial_{z}$ est majorée par le produit de $z_{0}$ par la coordonnée de $X$ sur $\partial_{y}$, ce qui permet de considérer le vecteur $X$ comme horizontal et d'interpoler entre $N\left(x_{i}\right)$ et $N$ dans le voisinage $U$. Les détails sont laissés au lecteur. 
La preuve de la proposition 2.1 se conclut en appliquant le lemme 2.3 ci-dessous.

Lemme 2.3 Si le lieu des contacts $\Delta$ d'une paire $\left(\xi_{+}, \xi_{-}\right)$possède un voisinage normal, alors $\left(\xi_{+}, \xi_{-}\right)$se laisse déformer en une paire normale.

Démonstration On considère un voisinage normal $N(\Delta)$ de $\Delta$. Il s'agit de modifier $\xi_{+}$et $\xi_{\text {- dans }} N(\Delta)$ pour obtenir une paire normale. Dans la suite, on se concentre sur un voisinage de $\Delta_{+}$. La construction au voisinage de $\Delta_{-}$est similaire.

Le lemme d'élimination des singularités de Giroux [6] permet de déformer $\xi_{+}$relativement à $V \backslash \operatorname{Int}(N(\Delta))$ pour que

- $\quad$ si $N_{j}, j=1, \ldots n$, est le voisinage d'une branche de foyers (resp. de selles), la structure $\xi_{+}$trace un foyer radial (resp. une selle) sur chaque disque horizontal de $N_{j}$

- sur chaque voisinage $N\left(x_{i}\right), i=1, \ldots, n$, la structure $\xi_{+}$trace sur le feuilletage par disques horizontaux de $N\left(x_{i}\right)$ le film d'une élimination, l'élimination se faisant le long des caractéristiques $\{x=0, z=c\}, c \in\left[-z_{0}, z_{0}\right]$.

La deuxième étape est de remplacer $\xi_{-}$à l'intérieur de $N(\Delta)$ par une structure de contact négative $\xi_{-}^{\prime}$ qui trace sur l'intérieur des disques horizontaux de $N(\Delta)$ le même feuilletage que $\xi_{+}$.

On commence par étendre $\left.\xi_{-}\right|_{V \backslash \text { Int } N(\Delta)}$ aux voisinages $N_{j}$ qui sont des branches de selles. Sur $N_{j}=[-1,1] \times[-1,1] \times[0, m]$, le feuilletage caractéristique $\xi_{+}\{z=c\} \mathrm{du}$ disque horizontal $[-1,1] \times[-1,1] \times\{c\}$ est donné par le noyau d'une $1-$ forme $\beta_{c}$ avec $\left.d \beta_{c}\right|_{[-1,1] \times[-1,1] \times\{c\}}>0$, qu'on peut supposer indépendante de $c$ sur un voisinage $U$ du bord vertical. On cherche à étendre $\xi_{-}$sur $N_{j}$ par le noyau d'une équation du type

$$
\alpha_{-}^{\prime}=d z-f \beta_{z}=0,
$$

où $f: N_{j} \rightarrow \mathbb{R}$ est strictement positive sur $\operatorname{Int}\left(N_{j}\right)$ et vérifie les conditions imposées par $\xi_{-}$au bord. La condition de contact est donnée par

$$
\left(d f \wedge \beta_{z}+f^{2} \beta_{z} \wedge \dot{\beta}_{z}+f d_{h} \beta_{z}\right) \wedge d z>0,
$$

où $\dot{\beta}_{z}$ est la dérivée de $\beta_{z}$ par rapport à $z$ et $d_{h}$ est la différentielle dans la direction horizontale.

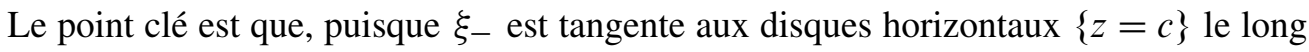
des $\operatorname{arcs}\{|y|=1, z=c\}$, la fonction $f$ est nulle le long de ces mêmes arcs. Elle peut donc être choisie arbitrairement petite et de différentielle nulle en dehors de $U$. Comme sa valeur est positive le long des arêtes $\{|x|=1, z=c\}$, on peut également imposer à sa 
dérivée dans la direction du feuilletage caractéristique de $\xi_{+}$d'être positive. Pour un tel choix de fonction $f$, la forme $\alpha_{-}^{\prime}$ est de contact: en dehors de $U, d f$ est nulle et $f^{2}$ est négligeable devant $f$, donc $\left(d f \wedge \beta_{z}+f^{2} \dot{\beta}_{z} \wedge \beta_{z}+f d_{h} \beta_{z}\right) \wedge d z$ est de l'ordre de $f d_{h} \beta_{z} \wedge d z>0$. Dans $U, \dot{\beta}_{z}=0$ et $d f \wedge \beta_{z} \wedge d z>0$, tout comme $f d_{h} \beta_{z} \wedge d z>0$. L'inégalité de contact est donc encore satisfaite.

On s'occupe ensuite de la partie $N_{f}$ de $N$ qui est un voisinage des branches de foyers. Une petite difficulté technique apparaît: les bords des disques $[-1,1] \times[-1,1] \times\{0\}$ et $[-1,1] \times[-1,1] \times\{m\}$ ont une arête legendrienne pour $\xi_{-}$. On commence donc par élargir $N_{f}$ en $N_{f}^{\prime}$ pour que $N_{f}^{\prime}$ soit feuilleté par des disques dont les bords sont transversaux positivement à $\xi_{+}$et négativement à $\xi_{-}$(et avec le bord vertical de $N_{f}^{\prime}$ transversal à $X$ ). On étend les coordonnées de $N_{f}$ à $N_{f}^{\prime}$ pour que $\xi_{+}$trace toujours un feuilletage radial sur chaque disque horizontal de $N_{f}^{\prime}$. On remplace alors la structure $\xi_{-}$ sur $N_{f}^{\prime}$ par une structure $\xi_{-}^{\prime}$ tangente aux feuilletages caractéristiques radiaux tracés $\operatorname{par} \xi_{+}$, en la faisant pivoter de sa position initiale au bord jusqu'à la position horizontale, atteinte au centre.

Reste à étendre $\xi_{-}$dans les voisinages $N\left(x_{i}\right), i=1, \ldots, n$, des contacts quadratiques. Dans chacun des voisinages $N\left(x_{i}\right)$, le feuilletage caractéristique tracé par $\xi_{+}$sur les disques horizontaux donne le film d'une élimination d'un foyer avec une selle. La remarque clé est, comme dans le cas des selles, qu'on peut alors étendre la restriction de $\xi_{-}$au bord vertical de $N\left(x_{i}\right)$ en une structure de contact qui trace sur l'intérieur de chacun des disques horizontaux le même feuilletage que $\xi_{+}$, et ce car $\xi_{-}$est tangente aux arcs $\left\{y=z_{0}+\sqrt{z_{0}}, z=c\right\}, c \in\left[-z_{0}, z_{0}\right]$. Ce procédé fournit une première structure qui ne coïncide pas nécessairement avec $\xi_{-}$le long des faces horizontales $\left\{z= \pm z_{0}\right\}$. Pour obtenir une structure qui étend $\xi_{-}$, on interpole entre cette première extension, d'équation $d z+\beta_{z}=0$, et $\xi_{-}$, d'équation $d z+\delta_{z}=0\left(\beta_{z}\right.$ et $\delta_{z}$ ont même noyau dans $\left.\operatorname{Int}\left(N\left(x_{i}\right)\right)\right)$, dans un voisinage de $\left\{z= \pm z_{0}\right\}$. On obtient un champ de plans avec une équation du type $d z+\chi(z) \beta_{z}+(1-\chi(z)) \delta_{z}=0$, où $\chi$ est une fonction de coupure, qui est une extension de $\xi_{-}$et qui va automatiquement être de contact.

Pour finir, on vérifie que la structure $\xi_{-}^{\prime}$ produite est isotope à la structure initiale $\xi_{-}$. Pour cela, on observe par exemple que les structures $\xi_{-}$et $\xi_{-}^{\prime}$ sont tendues sur $N(\Delta)$ (car "horizontales"). La structure $\xi_{-}^{\prime}$ est égale à $\xi_{-}$le long de $\partial N(\Delta)$ et toutes deux possèdent un cercle méridien transversal et d'autoenlacement -1 . L'isotopie est alors donnée par un avatar du théorème de classification d'Eliashberg [3] (il est également possible ici de trouver une isotopie explicite par des arguments élémentaires).

Remarque 2 La perturbation d'une paire générique $\left(\xi_{+}, \xi_{-}\right)$en une paire normale $\left(\xi_{+}^{\prime}, \xi_{-}^{\prime}\right)$ peut être réalisée en fixant $\Delta$. 


\subsection{Plans localement intégrables donnés par une paire normale}

Soit $\left(\xi_{+}, \xi_{-}\right)$une paire de structures de contact normale. On note toujours $X$ un champ de vecteurs lisse, nul sur $\Delta$ et qui dirige le champ de droites orientées $\xi_{+} \cap \xi_{-}$sur $V \backslash \Delta$. Comme dans [5], on peut alors définir quatre champs de plans $\lambda_{ \pm}^{ \pm \infty}$ en posant, pour $x \in V \backslash \Delta$ :

$$
\lambda_{ \pm}^{ \pm \infty}(x)=\lim _{t \rightarrow \pm \infty} X_{*}^{t}\left(X^{-t}(x)\right) \xi_{ \pm}\left(X^{-t}(x)\right) .
$$

Ici, $X^{t}$ désigne le flot de $X$ à l'instant $t$. On pose de plus, pour $x \in \Delta_{+}$,

$$
\lambda_{ \pm}^{+\infty}(x)=\xi_{+}(x)=\xi_{-}(x)
$$

et pour $x \in \Delta_{-}$,

$$
\lambda_{ \pm}^{-\infty}(x)=\xi_{+}(x)=\xi_{-}(x) .
$$

Proposition 2.4 Si $V$ est close, si $\left(\xi_{+}, \xi_{-}\right)$est une paire normale de structures de contact sur $V$ et si $\Delta_{-}$est transversale à $\xi_{+}$(s'il n'y a pas de contact quadratique le long de $\Delta_{-}$), alors $\lambda_{+}^{+\infty}=\lambda_{-}^{+\infty}=: \lambda^{+\infty}$ sur $V \backslash \Delta_{-}$.

Démonstration On reprend les notations de [5]. On fixe une métrique $g$ sur $V$ et on note $v$ le champ de plans orthogonal à $X$ sur $V \backslash \Delta$. On pose $\lambda_{ \pm}=\xi_{ \pm} \cap v$, et, pour $t \in \mathbb{R}$ et $x \in V \backslash \Delta$,

$$
\lambda_{ \pm}^{t}(x)=X_{*}^{t}\left(X^{-t}(x)\right) \xi_{ \pm}\left(X^{-t}(x)\right) \cap v(x) .
$$

On notera de la même façon la droite $\lambda_{ \pm}^{t}(x)$ et le plan contenant cette droite et le vecteur $X$. Si $\theta_{ \pm}^{t}(x)$ désigne l'angle (calculé avec $g$ ) entre $\lambda_{ \pm}^{t}(x)$ et $\lambda_{ \pm}(x)$, les conditions de contact imposent

$$
\frac{d \theta_{+}^{t}(x)}{d t}>0 \text { et } \quad \frac{d \theta_{-}^{t}(x)}{d t}<0 .
$$

Ces conditions, plus le fait que les fonctions $\theta_{ \pm}^{t}(x)$ soient bornées (à $x$ fixé, et indépendemment de $t$ ), suffisent pour assurer l'existence de limites quand $t$ tend vers $\pm \infty$. Il est alors automatique que les plans limites $\lambda_{ \pm}^{ \pm \infty}$ sont invariants par le flot de $X$.

On traite le cas où $t$ tend vers $+\infty$. On note $O$ l'orbite de $X$ qui passe par un point $x \in V \backslash \Delta$. Pour prouver la proposition 2.4 dans cette situation, on distingue six cas. Les deux premiers sont essentiellement traités dans [5]. Pour le confort du lecteur, on reproduit tout de même la preuve de ces deux cas spéciaux. La preuve proposée pour le cas 2 tient compte du fait qu'ici, contrairement à [5], l'espace $V \backslash \Delta$ n'est pas compact. Comme la paire considérée est normale, soit l'orbite $O$ a une limite $x_{-\infty}$ 
dans $\Delta$ lorsque $t$ tend vers $-\infty$, soit elle a un point d'accumulation en temps négatif dans $V \backslash \Delta$.

Cas $1 O$ est une orbite périodique.

On note $L: v \rightarrow v$ la différentielle de l'application de premier retour du flot de $X$ en $x$. Les plans $\lambda_{ \pm}^{+\infty}(x)$ et $\lambda_{ \pm}^{-\infty}(x)$ sont distincts et invariants par le flot de $X$. Si $\lambda_{-}^{+\infty} \neq \lambda_{+}^{+\infty}$, l'application linéaire $L$ a au moins trois directions propres distinctes et se trouve donc être une homothétie. Ceci contredit le fait que les directions $\lambda_{ \pm}$ de $\xi_{ \pm}$ne sont pas laissées invariantes par $L$. On peut également argumenter de la façon suivante, ce qui sera utile pour l'étude du cas 2 . Les directions $\lambda_{ \pm}^{+\infty}(x)$ sont des directions propres de $L$ et les droites $\lambda_{ \pm}(x)$ sont situées dans un même secteur de $v \backslash\left(\lambda_{+}^{+\infty}(x) \cup \lambda_{-}^{+\infty}(x)\right)$. Comme telles, ces dernières doivent être tournées dans le même sens par l'application linéaire $L$, ce qui n'est pas le cas, puisque $d \theta_{+}^{t}(x) / d t>0$ et $d \theta_{-}^{t}(x) / d t<0$.

Cas $2 O$ a une valeur d'adhérence en temps négatif dans $V \backslash \Delta$.

Dans ce cas, on fait fonctionner une version quantitative du cas 1). On suppose que $\lambda_{+}^{+\infty}(x) \neq \lambda_{-}^{+\infty}(x)$. Soit $x_{\infty} \in V \backslash \Delta$ la limite d'une suite $x_{n}=X^{t_{n}}(x)$ de points de $O$, avec $t_{n} \rightarrow-\infty$. Il existe $\alpha>0$ tel que, si $t$ est assez grand, $\theta_{+}^{t}\left(x_{\infty}\right) \geq \alpha$ et $\theta_{-}^{t}\left(x_{\infty}\right) \leq-\alpha$. On en déduit qu'il existe $\alpha>0$, un voisinage $U$ de $x_{\infty}$ et $T>0$, tels que pour tout $p \in U$ et pour tout $t \geq T,\left|\theta_{ \pm}^{t}(p)\right|>\alpha$. Quitte à prendre $n$ assez grand, on suppose que tous les $x_{n}$ sont dans $U$. On identifie $U$ à $\mathbb{R}^{3}$, en rendant $v$ tangent à $T\left(\{*\} \times \mathbb{R}^{2}\right)$ et $X$ colinéaire à $\mathbb{R} \times(*, *)$, si bien qu'on identifie tous les plans de $v$ par translation.

On suppose pour l'instant qu'on peut trouver une suite $\left(x_{\phi(n)}\right)$ extraite de $x_{n}$ telle que $\lambda_{+}^{+\infty}\left(x_{\phi(n)}\right)$ et $\lambda_{-}^{+\infty}\left(x_{\phi(n)}\right)$ aient pour limites respectives $\mu_{+}$et $\mu_{-}$, lorsque $n$ tend vers $+\infty$, avec $\mu_{+} \neq \mu_{-}$. Si $n$ et $p$ sont assez grands avec $n<p$, il existe dans ce cas une application linéaire $A_{n, p}: \mathbb{R}^{2} \rightarrow \mathbb{R}^{2}$ qui est $\epsilon$-proche de l'identité $(\epsilon \ll \alpha)$ et qui envoie $\lambda_{ \pm}^{+\infty}\left(x_{\phi(p)}\right)$ sur $\lambda_{ \pm}^{+\infty}\left(x_{\phi(n)}\right)$. Mais alors, dès que $t_{\phi(n)}-t_{\phi(p)}>T$, la composée de $A$ avec la différentielle $X_{*}^{t_{\phi(p)}-t_{\phi(n)}}\left(x_{\phi(n)}\right)$ a $\lambda_{ \pm}^{+\infty}\left(x_{\phi(n)}\right)$ comme espaces propres, et fait tourner les deux droites $\lambda_{ \pm}\left(x_{\phi(n)}\right)$, situées dans la même composante de $\mathbb{R}^{2} \backslash\left(\lambda_{+}^{+\infty}\left(x_{\phi(n)}\right) \cup \lambda_{-}^{+\infty}\left(x_{\phi(n)}\right)\right)$, dans des sens opposés (car $\left.\epsilon \ll \alpha\right)$, ce qui est impossible.

Il reste à étudier le cas où $\mu_{+}=\mu_{-}$. Cette fois, on peut trouver $n<p$ pour que les points $x_{\phi(n)}$ et $x_{\phi(p)}$ soient dans $U$ et que l'angle formé par les droites $\lambda_{+}^{+\infty}\left(x_{\phi(p)}\right)$ et $\lambda_{-}^{+\infty}\left(x_{\phi(p)}\right)$ soit très petit (quitte à prendre $p$ assez grand) devant l'angle formé par $\lambda_{+}^{+\infty}\left(x_{\phi(n)}\right)$ et $\lambda_{-}^{+\infty}\left(x_{\phi(n)}\right)$. On peut alors trouver une application linéaire (pas proche 
de l'identité) $A_{n, p}$ qui envoie les deux droites $\lambda_{ \pm}^{+\infty}\left(x_{\phi(p)}\right)$ sur $\lambda_{ \pm}^{+\infty}\left(x_{\phi(n)}\right)$ et qui "écarte" les droites $\lambda_{ \pm}\left(x_{\phi(p)}\right)$ : les droites $\lambda_{ \pm}\left(x_{\phi(n)}\right)$ sont dans un même secteur de

$$
\mathbb{R}^{2} \backslash\left(A_{n, p} \lambda_{+}\left(x_{\phi(p)}\right) \cup A_{n, p} \lambda_{-}\left(x_{\phi(p)}\right)\right) .
$$

Par les conditions de contact, le secteur angulaire délimité par les droites $\lambda_{+}\left(x_{\phi(n)}\right)$ et $\lambda_{-}\left(x_{\phi(n)}\right)$ est envoyé par la différentielle $X_{*}^{t_{\phi(p)}-t_{\phi(n)}}\left(x_{\phi(n)}\right)$ sur un secteur contenant les droites $\lambda_{ \pm}\left(x_{\phi(p)}\right)$. La composition de $A_{n, p}$ avec la différentielle $X_{*}^{t_{\phi(p)}-t_{\phi(n)}}\left(x_{\phi(n)}\right)$ a $\lambda_{ \pm}^{+\infty}\left(x_{\phi(n)}\right)$ comme espaces propres et fait comme précédemment tourner les droites $\lambda_{ \pm}\left(x_{\phi(n)}\right)$ en sens opposés. C'est la contradiction recherchée.

Cas $3 O$ provient d'un foyer de $\Delta_{+}$.

Dans ce cas, on peut modéliser un voisinage du foyer $e$ en question par

$$
\left.\left.\mathbb{R}^{3}=\{(r, \theta, z) \in] 0, \epsilon\right] \times \mathbb{R} / \mathbb{Z} \times[-1,1]\right\},
$$

$\xi_{+}=\left\{d z-f\left(r^{2}, \theta, z\right) d \theta=0\right\}, f(0, \theta, z)=0$ et $\xi_{-}=\left\{d z-g\left(r^{2}, \theta, z\right) d \theta=0\right\}$, $g(0, \theta, z)=0$. On vérifie alors que dans ces coordonnées, $\lambda \pm^{\infty}(r, \theta, z)=\{d z=0\}$. Cette égalité, obtenue explicitement au voisinage de $e$, se propage en $x$ par le flot de $X$ (de même que la différentiabilité de ce champ de plans limite).

Cas $4 O$ est la séparatrice instable d'une selle de $\gamma_{+}$.

On utilise un modèle comme dans le cas 3 , qui montre que le long de la séparatrice instable, au voisinage de la selle $h$, les plans limites $\lambda_{ \pm}^{+\infty}$ coïncident avec le plan tangent au feuilletage par disques qui contient $X$. Précisemment, si on conjugue un voisinage de $h$ à sa linéarisation, on obtient, dans des coordonnées $(a, b, c) \in D^{2} \times[-\epsilon, \epsilon]$, que le flot de $X$ est $X^{t}(a, b, c)=\left(a \exp \left(\mu_{+} t\right), b \exp \left(\mu_{-} t\right), c\right)$. Dans ce modèle, $\mu_{+}>-\mu_{-}>0$ car $h$ est à divergence positive et $x$ est sur la séparatrice $s$ qui est $\{a>0, b=c=0\}$ près de $h$. Les plans de contact $\xi_{+}$et $\xi_{-}$ont quant à eux pour équations le long de $s$ respectivement $d c+\alpha a d b=0$ et $d c-\beta a d b=0$. On calcule alors que $X_{*}^{t}\left(X^{-t}(a, 0,0)\right) \xi_{+}\left(X^{-t}(a, 0,0)\right)$ est d'équation $d c+\alpha \exp \left(\left(-\mu_{+}-\mu_{-}\right) t\right) d b=0$. Lorsque $t$ tend vers l'infini la limite est bien $\{d c=0\}$. On aboutit au même résultat pour $\xi_{-}$.

Comme dans le cas précédent, l'égalité $\lambda_{+}^{+\infty}=\lambda_{-}^{+\infty}$ se prolonge en $x$ par le flot de $X$.

Cas $5 O$ est la séparatrice instable d'une selle de $\Delta_{-}$.

Dans ce cas, $\lambda_{ \pm}^{+\infty}$ est le plan tangent à la réunion des variétés instables issues de $\Delta_{-}$. On démontre ce fait en reprenant le modèle local du cas 4 ), avec cette fois $-\mu_{-}>\mu_{+}>0$.

Cas $6 O$ provient d'une naissance-mort de $\Delta_{+}$. 
Si $O$ provient de $\Delta_{+}$, et de l'intérieur de la partie foyer de la naissance-mort, le plan $\lambda_{ \pm}^{+\infty}$ est à nouveau, au voisinage de la singularité, le plan tangent au feuilletage en disques, donné par la forme normale, qui contient $X$. Un germe de feuille intégrale passant par $x$ est en particulier lisse, comme dans le cas des foyers. Si $O$ est l'une des deux orbites bord de la partie foyer, l'écriture d'un modèle local fournit également l'égalité recherchée.

Proposition 2.5 Si $\left(\xi_{+}, \xi_{-}\right)$est une paire normale sur une variété close $V$ et si $\Delta_{-}$ est transversal à $\xi_{+}$, alors le champ de plans $\lambda^{+\infty}$ est continu sur $V \backslash \Delta_{-}$, invariant par le flot de $X$ et localement intégrable. Lorsque de plus $\Delta_{+}$est transversal à $\xi_{+}$, alors les deux champs de plans $\lambda^{+\infty}$ et $\lambda^{-\infty}$ (défini de façon similaire à $\lambda^{+\infty}$ ) sont transversaux en dehors de $\Delta$.

En particulier par tout point où $\lambda^{ \pm \infty}$ est défini passe un germe de variété intégrale. Ce germe n'est pas forcément unique, et $\lambda^{ \pm \infty}$ ne possède pas forcément de feuilletage intégral global.

Démonstration On montre que $\lambda^{+\infty}$ est continu sur $V \backslash \Delta_{-}$. On note $\theta_{+}^{\prime t}(x)=$ $\theta_{+}^{t}(x), \theta_{-}^{\prime t}(x)$ l'angle entre $\lambda_{-}^{t}(x)$ et $\lambda_{+}(x)$, et $\theta^{\prime \infty}$ la limite commune de $\theta_{+}^{\prime t}$ et $\theta_{-}^{\prime t}$ quand $t$ tend vers $+\infty$. Comme $\theta_{-}^{t}(x)$, la fonction $\theta_{-}^{\prime t}(x)$ est une fonction décroissante de $t$.

Soit $\epsilon>0$ et $x \in V \backslash \Delta$. Il existe $t_{0}$ tel que, pour $t \geq t_{0}$,

$$
\theta^{\prime \infty}(x)-\epsilon<\theta_{-}^{\prime t}(x)<\theta_{+}^{\prime t}(x)<\theta^{\prime \infty}(x)+\epsilon
$$

(pour des relèvements convenablement choisis des angles $\theta^{\prime}$ dans $\mathbb{R}$ ). Par continuité $\mathrm{du}$ flot de $X$ par rapport à $x$, si $y$ est assez proche de $x$, on a $\left|\theta_{ \pm}^{\prime t_{0}}(y)-\theta_{ \pm}^{\prime t_{0}}(x)\right|<\epsilon / 2$. Mais alors, pour $t \geq t_{0}, \theta^{\prime \infty}(x)-3 \epsilon / 2<\theta_{-}^{\prime t_{0}}(y)<\theta_{-}^{\prime t}(y)<\theta^{\prime \infty}(y)<\theta_{+}^{\prime t}(y)<$ $\theta_{+}^{\prime t_{0}}(y)<\theta^{\prime \infty}(x)+3 \epsilon / 2$. On en déduit la continuité de $\theta^{\prime \infty}$, et donc de $\lambda^{+\infty}$, sur $V \backslash \Delta$.

On a également bien évidemment continuité aux points de $\Delta_{+}$, car le plan $\lambda^{+\infty}$ est coincé entre $\xi_{+}$et $\xi_{-}$qui tendent tous deux vers le même plan. C'est donc aussi le cas de $\lambda^{+\infty}$.

On montre maintenant que $\lambda^{+\infty}$ est localement intégrable: par tout point de $V \backslash \Delta_{-}$ passe un germe de surface intégrale.

Si $x \in V \backslash \Delta$, on se donne un germe de disque $D$ centré en $x$ et transversal à $X$. Le champ de plans $\lambda^{+\infty}$ intersecte le plan tangent à $D$ en un champ de droites continu. Par le théorème de Cauchy et Péano, ce champ de droites admet un germe de courbe 
intégrale $\gamma$ passant par $x$. Le germe de surface obtenu en poussant $\gamma$ par le flot de $X$ est tangent à $\lambda^{+\infty}$, puisque $X$ est tangent à $\lambda^{+\infty}$ et que $\lambda^{+\infty}$ est invariant par le flot de $X$.

Si $x$ est un foyer de $\Delta_{+}$, le modèle local donné dans la preuve de la proposition 2.4 donne que $\lambda^{+\infty}$ est lisse et uniquement intégrable au voisinage de $x$ (c'est le champ de plans horizontal).

Si $x$ est une selle de $\Delta_{+}$, on se replace dans les coordonnées de la proposition 2.4, où le flot de $X$ est $(a, b, c) \rightarrow\left(a \exp \left(\mu_{+} t\right), b \exp \left(\mu_{-} t\right), c\right)$ au temps $t$ dans un voisinage $U$ de $x$, et $x=(0,0,0)$. On se donne deux germes de disques $D_{1} \subset U$ et $D_{2} \subset U$ transversaux à $X$ et intersectant les deux séparatrices stables de $x$. On choisit ensuite à l'aide du théorème de Cauchy et Péano, pour $i=1,2$, des courbes intégrales $\gamma_{i} \subset U$ de $T D_{i} \cap \lambda^{+\infty}$ passant par ces séparatrices. On considère alors l'ensemble $S$ des trajectoires de $\left.X\right|_{U}$ issues de $\gamma_{1}$ et $\gamma_{2}$, augmenté des séparatrices instables de $x$ dans $U$. On montre que $S$ donne un germe de surface intégrale de $\lambda^{+\infty}$ près de $X$. Tout d'abord, $S$ est le graphe d'une fonction $f$ continue: $S=\{(a, b, f(a, b))\}$ près de $x$. Il est automatique que les ensembles $S_{1}$ et $S_{2}$ des trajectoires issues respectivement de $\gamma_{1}$ et $\gamma_{2}$ forment deux surfaces intégrales ouvertes de $\lambda^{+\infty}$. Il faut voir que ces deux surfaces se recollent le long des séparatrices instables de $x$ pour former une surface intégrale globale. Pour cela, on utilise le modèle local: une orbite issue d'un point $(a, b, \epsilon)$ passe en $\left(a^{\prime}, b\left(a / a^{\prime}\right)^{-\mu_{-} / \mu_{+}}, \epsilon\right)$, avec $(b / a)\left(a / a^{\prime}\right)^{-\mu_{-} / \mu_{+}} \rightarrow+\infty$ quand $a \rightarrow 0$, car $0<-\mu_{-} / \mu_{+}<1$. Ceci permet de majorer le taux de variation de $f$ en $\left(a^{\prime}, 0\right)$ par celui en un point $(0, b)$ où $f$ est différentiable et de différentielle nulle. On obtient que $f$ est différentiable de différentielle nulle en $\left(a^{\prime}, 0\right)$, ce qui confirme que $S$ possède un plan tangent égal à $\lambda^{+\infty}$ le long des séparatrices instables de $x$.

On traite de manière similaire le cas où $x$ est une naissance-mort: on a déjà une demi-surface intégrale du côté foyer, qui se recolle avec une surface intégrale ouverte saturée par $X$ du côté selle, comme dans le sous-cas précédent (même si cette fois le modèle local ne donne pas une convergence vers $+\infty$, mais de façon suffisante un quotient de la deuxième coordonnée par $a$ minoré par une constante $>0$ ).

\section{Paires positives de structures de contact}

On suppose maintenant que $\Delta_{-}=\varnothing$. La paire de contact $\left(\xi_{+}, \xi_{-}\right)$est alors dite positive. On rappelle qu'on ne considère que des paires normales. Pour celles-ci, on s'intéresse au champ de plans $\lambda^{+\infty}$ qui est continu et défini sur tout $V$. On le note $\lambda$ pour simplifier les notations. On possède de nombreux exemples de telles paires de structures de contact positives. Un cas important se trouve dans [5]: tout feuilletage $\mathcal{F}$, 
différent du feuilletage en sphères de $S^{1} \times S^{2}$, peut être approché par des structures de contact positives et négatives. Un tel couple de structures de contact proches de $\mathcal{F}$ est automatiquement positif et se laisse déformer en une paire normale et positive d'après la proposition 2.1.

\subsection{Interactions paires/feuilletage}

On rappelle qu'un feuilletage $\mathcal{F}$ de codimension 1 sur une variété de dimension trois est tendu s'il possède une transversale qui coupe toutes ses feuilles. L'existence d'une telle transversale implique l'absence de composante de Reeb.

Lemme 3.1 $S i\left(\xi_{+}, \xi_{-}\right)$est une paire normale et positive sur une variété close $V$, les champs de plans $\xi_{ \pm}^{t}=X_{*}^{t} \xi_{ \pm}$convergent uniformément vers $\lambda$ lorsque $t$ tend vers $+\infty$.

Démonstration Les fonctions $\theta_{ \pm}^{t}(x)$ sont monotones en $t$ et convergent simplement vers une limite continue. Comme la variété $V$ est compacte, cette convergence est uniforme d'après le théorème de Dini.

Proposition 3.2 Si $\left(\xi_{+}, \xi_{-}\right)$est une paire normale positive et si $\xi_{+}$ou $\xi_{-}$est tendue, alors $\lambda$ ne possède pas de sphère intégrale.

Démonstration Soit $e\left(\xi_{ \pm}\right) \in H^{2}(V, \mathbb{Z})$ la classe d'Euler de $\xi_{ \pm}$. Si $S$ est une sphère intégrale de $\lambda$, alors le feuilletage caractéristique de $S$ pour $\xi_{+}$et $\xi_{-}$ne possède que des singularités positives. On en déduit que $e\left(\xi_{ \pm}\right) .[S]>0$. Or pour une structure $\xi$ tendue, on a $e(\xi) .[S]=0$ d'après $[1 ; 3]$.

Remarque 3 Si $S$ est une surface intégrale de $\lambda$, même si $S$ n'est pas lisse, son feuilletage caractéristique est bien défini puisque dirigé par le champ de vecteurs lisse $X$.

Théorème 3.3 Si $\left(\xi_{+}, \xi_{-}\right)$est une paire normale positive sur une variété close $V$, si $\xi_{+}$et $\xi_{-}$sont tendues et si $\lambda$ est uniquement intégrable, alors le feuilletage intégral $\mathcal{F}$ de $\lambda$ ne possède pas de composante de Reeb d'âme homologue à zéro dans $H_{1}(V, \mathbb{Q})$. De plus, $V$ porte un feuilletage sans composante de Reeb et en particulier son revêtement universel est $\mathbb{R}^{3}$.

Remarque 4 Réciproquement, si $\lambda$ a pour feuilletage intégral un feuilletage tendu $\mathcal{F}$, les structures $\xi_{ \pm}$sont tendues (et même symplectiquement remplissables), puisque isotopes à des structures proches de $\mathcal{F}$ (lemme 3.1) et donc soumises aux conclusions de [5]. 
Démonstration Soit $\mathcal{F}$ le feuilletage intégral de $\lambda$. On suppose que $\mathcal{F}$ contient une composante de Reeb, c'est-à-dire en particulier une feuille torique $T$ qui borde un tore solide.

On se donne un méridien $m$ tracé sur $T$ et une bande $B$ d'âme $m$, transversale à $\mathcal{F}$. La remarque clé est que si l'holonomie de $\mathcal{F}$ le long de $m$ est non triviale (si son germe n'est pas l'identité), on peut tracer sur $B$ une courbe plongée $\gamma$ positivement transversale à $\mathcal{F}$ et isotope à $m$ dans $B$. Le feuilletage tracé par $\mathcal{F}$ sur $B$ n'étant que $C^{0}$, pour trouver cette transversale, on utilise ici de manière cruciale que le champ de droites continu $T \mathcal{F} \cap T B$ est uniquement intégrable, conjugué à un résultat classique de Kneser (voir par exemple le "théorème de Kneser" dans le livre [11]). Cette courbe $\gamma$ est le bord d'un disque plongé dans $V$.

Comme $\xi_{ \pm}^{t}$ converge uniformément vers $\lambda$ lorsque $t$ tend vers $+\infty$, il existe $t_{0}$ tel que si $t \geq t_{0}$, les structures $\xi_{ \pm}^{t}$ soient transversales à $\gamma$, positivement pour les deux. On en déduit une homotopie entre $\xi_{+}^{t_{0}}$ et $\xi_{-}^{t_{0}}$ parmi les champs de plans transversaux à $\gamma$. Elle est construite en concaténant le chemin entre $\xi_{+}^{t_{0}}$ et $\lambda$ donné par les $\xi_{+}^{t}, t \geq t_{0}$, et le chemin entre $\lambda$ et $\xi_{-}^{t_{0}}$ donné par les $\xi_{-}^{t}, t \geq t_{0}$.

Cette homotopie indique que les autoenlacements de $\gamma$ pour les structures $\xi_{+}^{t_{0}}$ et $\xi_{-}^{t_{0}}$ sont opposés (les structures sont de signes opposés). On ne peut donc pas avoir pour les deux structures l'inégalité de Bennequin [1]: $l(\gamma) \leq-1$. Une au moins des structures est vrillée. Par contradiction, l'holonomie de $\mathcal{F}$ le long de $m$ est l'identité.

Soit à présent $l$ une longitude tracée sur $T$. On montre que si $[l]$ est nulle dans $H_{1}(V, \mathbb{Q})$, alors le germe de l'holonomie de $\mathcal{F}$ le long de $l$ est l'identité au moins d'un côté de $T$.

Si tel n'est pas le cas, même lorsque $l$ n'est pas homologue à zéro, on peut trouver un petit voisinage tubulaire $T \times[-1,1]$ de $T, T=T \times\{0\}$, où les tores $T \times\{ \pm 1\}$ sont transversaux à $\mathcal{F}$. Comme l'holonomie de $\mathcal{F}$ le long de $m$ est l'identité, on a de plus que les feuilletages tracés par $\mathcal{F}$ sur $T \times\{ \pm 1\}$ sont des feuilletages en cercles méridiens.

À ce stade, on peut utiliser le fait, dû à Haefliger [10], que l'union des feuilles compactes de $\mathcal{F}$ est un compact de $V$ pour considérer les feuilles toriques maximales parmi celles qui bordent un tore solide (la maximalité se rapporte à l'inclusion des tores solides). D'après ce qui précède, un tel tore maximal est inclus dans un tore solide sur le bord duquel $\mathcal{F}$ trace un feuilletage par cercles méridiens. On peut remplacer $\mathcal{F}$ sur ce tore par un feuilletage en disques. Cette construction conduit à un feuilletage $\mathcal{F}^{\prime}$ sans composante de Reeb. 
Pour revenir à l'étude du feuilletage $\mathcal{F}$ dans le cas où $[l]=0$, on note que, comme $\xi_{+}^{t}$ converge uniformément vers $\mathcal{F}$, pour $t$ assez grand, $\xi_{+}^{t}$ est transversal à $T \times\{ \pm 1\}$ et le feuilletage caractéristique $\xi_{+}^{t} T \times\{ \pm 1\}$ est non singulier et arbitrairement proche d'un feuilletage par cercles méridiens. Le feuilletage caractéristique de $T$ pour $\xi_{+}^{t}$ est lui constant et dirigé par $X$. Il possède une certaine pente $p$ (bien définie modulo $\pi$ par le choix d'une longitude fixe $l$ de $T$ ), différente de celle du méridien (qui vaudra par définition 0) car sinon on obtiendrait un disque vrillé. Cette pente est la pente de n'importe quelle courbe intégrale de son feuilletage caractéristique, car celui-ci ne possède que des singularités positives.

On note $p_{+}^{ \pm 1, t}$ les pentes de $\xi_{+}^{t} T \times\{ \pm 1\}$. Si $t$ est assez grand, elles sont non nulles (car sinon $\xi_{+}$serait vrillée), dépendent continûment de $t$ et tendent vers 0 (modulo $\pi$ ) lorsque $t$ tend vers $+\infty$. On peut alors également les supposer irrationnelles.

Si $t$ est assez grand, les pentes $p_{1}^{1, t}$ et $p_{1}^{-1, t}$ sont proches de 0 et en particulier, elles délimitent un secteur angulaire de petite ouverture qui ne contient pas $p$. Si on parcourt le cercle dans le sens trigonométrique pour joindre $p_{1}^{1, t}$ à $p_{1}^{-1, t}$ en passant par $p$, on est alors assuré de traverser un intervalle $I(t)$ de taille minorée par $\pi-\epsilon(t)$, où $\epsilon(t)$ tend vers 0 quand $t$ tend vers $+\infty$.

La restriction de $\xi_{+}^{t}$ a $T \times[-1,1]$ est universellement tendue: proche de $\mathcal{F}$, elle est transversale à une fibration en intervalles de $T \times[-1,1]$; son rappel dans le revêtement universel de ce produit se plonge alors de manière explicite dans la structure de contact tendue standard de $\mathbb{R}^{3}$. D'après le théorème principal de [7] qui classifie les structures de contact tendues sur le tore épais, pour chaque pente $p^{\prime}$ dans l'intervalle $I(t)$, il existe un tore $T_{p^{\prime}}$ dans $T \times[-1,1]$, isotope à $T$ et dont le feuilletage caractéristique pour $\xi_{+}^{t}$ est non singulier et de pente $p^{\prime}$. En particulier $I(t)$ ne contient pas 0 et $\pi$ (modulo $2 \pi$ ), car sinon $\xi_{+}^{t}$, et donc $\xi_{+}$, serait vrillée. On note $k$ l'ordre de l'âme de la composante de Reeb dans $H_{1}(V, \mathbb{Z})$. Pour tout $n \in \mathbb{Z}$ premier avec $k$, si $t$ est assez grand, toute courbe de pente $k / n$ est réalisée par une courbe non singulière $\gamma_{k / n}$ du feuilletage caractéristique pour $\xi_{+}^{t}$ d'un tore $T_{k / n}$. Soit $n \in \mathbb{Z}$ premier avec $k$. Pour tout $l \in \mathbb{Z}$, la courbe $\gamma_{k /(n+l k)}$ est obtenue comme image de $\gamma_{k / n}$ par la puissance $l$-ième d'un twist de Dehn autour du méridien. Comme courbe lisse, elle est isotope à $\gamma_{k / l}$. Elle borde donc une surface compacte et orientée de genre $g$ indépendant de $l$. Son invariant de Thurston-Bennequin dépend affinement de $l$ : la différence entre $\operatorname{tb}\left(\gamma_{k /(n+l k)}\right)$ et $\operatorname{tb}\left(\gamma_{k} /(n+(l+1) k)\right)$ vaut $k$, et donc l'inégalité de Bennequin

$$
\operatorname{tb}\left(\gamma_{k /(n+l k)}\right) \leq 2 g-1
$$

sera violée pour un choix de $l$ judicieux.

On en déduit par contradiction que l'holonomie est l'identité au moins d'un côté de $T$. Cette argument montre également que si $U \simeq T^{2} \times[0,1]$ est une sous-variété 
de $V$ feuilletée par des tores $\left(T^{2} \times\{s\}\right)_{s \in[0,1]}$ de $\mathcal{F}$, alors l'holonomie de $\mathcal{F}$ est l'identité près de $T^{2} \times\{0\}$ ou de $T^{2} \times\{1\}$. Comme on est parti d'un tore $T$ bordant une composante de Reeb, et en particulier un tore solide, on en déduit que $V$ est difféomorphe à $S^{1} \times \mathbb{R}^{2}$, ce qui donne une contradiction.

Remarque $5 \mathrm{Si}\left(\xi_{+}, \xi_{-}\right)$est une paire positive de structures de contact tendues, l'inégalité de Bennequin montre, même lorsque $\lambda$ n'est pas uniquement intégrable, qu'aucune courbe transversale à $\lambda$ n'est le bord d'un disque plongé dans $V$.

Sous les conditions du théorème 3.3, le feuilletage $\mathcal{F}$ peut-il posséder des composantes de Reeb?

L'hypothèse de positivité faite sur la paire n'est pas très agréable à manipuler. On pourrait essayer de la remplacer par une condition d'homotopie: une paire de structures de contact $\left(\xi_{+}, \xi_{-}\right)$tendues qui sont homotopes comme champs de plans est-elle toujours déformable en une paire positive? Autrement dit, si $\xi_{+}$et $\xi_{-}$sont tendues et homotopes, peut-on toujours éliminer leurs contacts négatifs par une isotopie de $\xi_{+}$? Par ailleurs, il y a des paires positives de structures de contact, même transversales, pour lesquelles $\lambda$ n'a pas de feuilletage intégral global $[5 ; 13 ; 14]$. La notion de paire positive de structures de contact tendues semble généraliser strictement celle de feuilletage sans composante de Reeb. Quelles sont les variétés qui portent une telle paire? Parmi celles-ci, y en a-t-il une qui ne porte pas de feuilletage sans composante de Reeb?

Ces commentaires et le théorème 3.3 nous incitent à formuler la conjecture suivante.

Conjecture Si $V$ est une variété close qui porte une paire de structures tendues homotopes, alors le revêtement universel de $V$ est conjugué à $\mathbb{R}^{3}$.

Pour ce qui concerne les structures de contact universellement tendues sur les variétés irréductibles, la preuve de cette conjecture résulte du fait que la sphère $S^{3}$ ainsi que le produit $S^{2} \times \mathbb{R}$ ne portent pas de structures de contact tendues positives et négatives homotopes [3]. L'utilisation du théorème d'uniformisation démontré par Perelman [15] permet d'exclure toute autre possibilité que $\mathbb{R}^{3}$.

Soit $V$ une variété compacte de bord non vide portant une paire positive $\left(\xi_{+}, \xi_{-}\right)$. On dit que le bord de $V$ est adapté à la paire $\left(\xi_{+}, \xi_{-}\right)$si $\Delta=\Delta_{+}$est transversale à $\partial V$ et si $X$ est transversal à $\partial V$ et sort de $V$ en dehors des points de $\Delta \cap \partial V$. C'est par exemple le cas lorsque $\partial V$ est composé de tores transversaux à $X$ (et n'intersectant pas $\Delta$ ) ou de sphères intersectant $\Delta$ en deux foyers, comme dans le théorème 4.1. Dans la situation où le bord de $V$ est adapté à une paire positive $\left(\xi_{+}, \xi_{-}\right)$, on obtient, comme dans le cas clos, l'existence d'un champ de plans $\lambda=\lambda^{+\infty}$, continu et localement intégrable. Les théorèmes précédents s'étendent ainsi sans difficulté aux variétés compactes de bords adaptés à une paire $\left(\xi_{+}, \xi_{-}\right)$. 


\subsection{Un critère d'intégrabilité}

On donne maintenant un critère qui permet d'assurer que le champ de plans $\lambda$ est uniquement intégrable. Une paire positive de structures de contact est dite transitive si, pour tout $x \in V$, il existe $x_{-\infty} \in \Delta_{+}$tel que $X^{t}(x)$ converge vers $x_{-\infty}$ lorsque $t$ tend vers $-\infty$. Cette définition présente surtout un intérêt dans le cas où $V$ possède un bord non vide adapté à $\left(\xi_{+}, \xi_{-}\right)$.

Théorème 3.4 Soit $V$ une variété compacte dont le bord est adapté à une paire normale et positive $\left(\xi_{+}, \xi_{-}\right)$. Si la paire $\left(\xi_{+}, \xi_{-}\right)$est transitive et si le champ $X$ possède la propriété générique de n'avoir ni liaison entre les séparatrices d'une naissance-mort et d'une selle, ni suite de deux connections consécutives entre selles, alors le champ de plans $\lambda$ est uniquement intégrable.

Démonstration On observe que $\lambda$ est uniquement intégrable en un point $x$ de $V \backslash \Delta_{+}$ si et seulement si le champ de droites $\lambda \cap v$ est uniquement intégrable, où $v$ est le plan tangent à un germe de disque en $x$ transversal à $X$. (Pour obtenir le feuilletage intégral de $\lambda$, on pousse celui de $\lambda \cap v$ par le flot de $X$.) Si un point $x$ de $V$ est dans le bassin d'attraction d'un foyer, on a vu dans la preuve de la proposition 2.4 que le champ de plans $\lambda$ est $C^{\infty}$ au voisinage de $x$ et donc uniquement intégrable.

On suppose à présent que $x$ est sur la séparatrice instable d'une selle $h$. Par hypothèse de généricité, on est sûr qu'au moins une des deux séparatrices stable de $h$ provient d'un foyer $e$.

On étudie d'abord le cas où les deux séparatrices stables de $h$ proviennent de foyers $e$ et $e^{\prime}$. On fixe un petit disque $D$ passant par $x$ et transversal à $X$. Si $\lambda$ n'est pas uniquement intégrable en $x$, alors le champ de droites orientées $\lambda \cap T D$, dirigé par un vecteur $Y$, non plus. On peut donc trouver un petit $\operatorname{arc} \gamma$ inclus dans $D$ et transversal à $Y$ dont tous les points peuvent être obtenus en suivant $Y$ à partir de $x$ pendant un petit temps positif. Quitte à prendre $\gamma$ assez proche de $x$, on obtient également que tous les points de $\gamma$ proviennent de foyers proches de $e$ situés sur une même branche de $\Delta_{+}$en suivant le flot de $X$. En particulier, $\lambda$ est $C^{\infty}$ au voisinage de $\gamma$ et s'intègre uniquement sur un voisinage $U$ de $\gamma$ en un feuilletage en disques transversal à $\gamma$.

Si $y$ et $z$ sont deux points assez proches situés sur $\gamma$, ils doivent être dans le bassin d'attraction de deux foyers distincts $e_{0}$ et $e_{1}$ proches de $e$ (et situés sur la même composante de $\Delta_{+}$), sinon $y$ et $z$ seraient sur une même feuille du feuilletage intégral de $\left.\lambda\right|_{U}$. De ces deux foyers sont issues les séparatrices stables de deux selles $h_{0}$ et $h_{1}$ proches de $h$ (et situées sur la même composante de $\Delta_{+}$). Mais alors les feuilles intégrales de $\lambda$ passant par $y$ et $z$, qui sont bien définies dans les bassins d'attractions 
de $e_{0}$ et $e_{1}$, sont asymptotes aux séparatrices instables de $h_{0}$ et de $h_{1}$, ce qui exclut qu'elles puissent être asymptotes toutes les deux à la séparatrice de $h$.

Si on répète cet argument pour les petits temps négatifs de $Y$, on obtient que $Y$ est uniquement intégrable au voisinage de $x$, et donc aussi $\lambda$ (on rappelle que l'unique feuilletage intégral de $\lambda$ est obtenu en poussant le feuilletage intégral de $Y$ par le flot de $X$ qui est $C^{\infty}$ ).

Dans le cas où une séparatrice stable de $h$ provient d'un foyer et l'autre d'une selle $h^{\prime}$, on est sûr que (par généricité) les deux séparatrices stables de $h^{\prime}$ proviennent d'un foyer. On peut alors appliquer le raisonnement précédent le long des deux séparatrices instables de $h^{\prime}$ pour obtenir que $\lambda$ y est uniquement intégrable, et donc également au voisinage de celles-ci.

Reste le cas où la deuxième séparatrice stable de $h$ provient d'une naissance-mort $n$, génériquement de l'intérieur de la partie foyer. Si $\lambda$ n'était pas uniquement intégrable près de $x$, on trouverait une petite transversale $\gamma$ dont tous les points peuvent être atteints depuis $x$ par des petits arcs tangents à $Y$. Un petit sous-arc $\gamma^{\prime}$ de $\gamma$ serait constitué de points issus d'un petit arc de foyers (soient proches de $n$, soient proches du foyer dont provient génériquement la séparatrice stable de $n$ ). De ces foyers sont issues les séparatrices stables de selles proches de $h$. On raisonne alors comme dans le premier cas pour conclure. Le champ $\lambda$ est donc uniquement intégrable au voisinage des séparatrices stables de $h$.

L'argument développé dans le dernier sous-cas fournit également que si l'orbite d'un point $x$ provient de l'intérieur de la partie foyer d'une naissance-mort $n$, alors $\lambda$ est uniquement intégrable au voisinage de $x$. Le cas où l'orbite de $x$ est issue du bord de la partie foyer d'une naissance-mort se traite de manière identique à celle des séparatrices instables des selles. (On est dans la situation générique où la séparatrice stable de la naissance-mort provient d'un foyer.) Enfin, le cas où $x \in \Delta_{+}$(essentiellement si $x$ est une selle ou une naissance-mort, puisque celui des foyers est déjà traité) s'étudie par des méthodes similaires.

Le résultat suivant donne un moyen, par isotopie de $\xi_{+}$et $\xi_{-}$, de modifier l'ensemble des contacts positifs $\Delta_{+}$. Il pourrait permettre dans certains cas de rendre une paire transitive. C'est une version du lemme de création/élimination de singularités de [6] pour les paires.

Proposition 3.5 Soient $\left(\xi_{+}, \xi_{-}\right)$une paire de structures de contact, $\gamma$ une courbe fermée positivement transversale à $\xi_{+}$et $\xi_{-}$. On suppose que $\gamma \cap \Delta=\varnothing$ et qu'un voisinage $U \simeq \gamma \times D^{2}$ de $\gamma \simeq \gamma \times\{0\}$ est tel que $\xi_{+} \cap \xi_{-}=\xi_{+}\{*\} \times D^{2}=\xi_{-}\{*\} \times D^{2}$. 
Alors, il existe une isotopie de $\xi_{+}$et une isotopie de $\xi_{-}$à supports dans $U$ menant à une paire $\left(\xi_{+}^{\prime}, \xi_{-}^{\prime}\right)$ telle que $\xi_{+}^{\prime} \cap \xi_{-}^{\prime}=\xi_{+}^{\prime}\{*\} \times D^{2}=\xi_{-}^{\prime}\{*\} \times D^{2}$ et que les feuilletages caractéristiques $\xi_{+}^{\prime}\{*\} \times D^{2}$ et $\xi_{-}^{\prime}\{*\} \times D^{2}$ soient tous les deux égaux à un même feuilletage présentant une selle et un foyer positifs en position d'élimination.

Remarque 6 Dans cette proposition, le lieu des contacts positifs est modifié par l'ajout de deux composantes "parallèles", l'une formée de la réunion des selles, l'autre de la réunion des foyers inclus dans $U$.

Démonstration Soit $D_{\epsilon}^{2} \subset D^{2}$. On dessine sur $D_{\epsilon}^{2}$ un feuilletage de divergence positive constitué d'une selle et d'un foyer positif en position d'élimination. Il est défini comme noyau d'une 1 -forme $\beta$ avec $d \beta>0$. On munit $S^{1} \times D_{\epsilon}^{2}$ de la paire de structures de contact $\zeta_{ \pm}$d'équations $d t \pm \epsilon^{\prime} \beta=0, t \in S^{1}$. On dépose cette paire sur un voisinage tubulaire $\epsilon$-petit $U_{\epsilon} \simeq \gamma \times D_{\epsilon}$ de $\gamma, D_{\epsilon} \subset D$, identifié à $S^{1} \times D_{\epsilon}^{2}$. On fait en sorte $\zeta_{+} \cap \zeta_{-}=\xi_{+} \cap \xi_{-}$le long de $\partial U_{\epsilon}$. Il est alors possible [6], pour $\epsilon^{\prime}$ et $\epsilon$ assez petits, de raccorder $\zeta_{+}$à $\xi_{+}$et $\zeta_{-}$à $\xi_{-}$par une paire de structures transversales à $\partial / \partial t$ et à $T\left(\{*\} \times D^{2}\right)$ sur $U \backslash U_{\epsilon}$ - et donc transversales entre elles et dont l'intersection est dans $T\left(\{*\} \times D^{2}\right)$. La paire obtenue par recollement est la paire $\left(\xi_{+}^{\prime}, \xi_{-}^{\prime}\right)$ recherchée. Les structures $\xi_{ \pm}^{\prime}$ et $\xi_{ \pm}$sont isotopes comme dans [6].

\subsection{Paires fortement tendues}

Au lieu de s'intéresser aux paires positives de structures de contact tendues, on peut s'intéresser aux paires pour lesquelles deux points quelconques de $V$ peuvent être joints par un arc positivement transversal à $\xi_{+}$et $\xi_{-}$(et donc en particulier par un arc positivement transversal à $\lambda$, ce qui mime la définition de feuilletage tendu). Celles-ci sont automatiquement positives. On parlera alors de paire fortement tendue. Dans ce cas, on peut simplifier $\Delta_{+}$.

Proposition 3.6 Si $\left(\xi_{+}, \xi_{-}\right)$est une paire normale et fortement tendue, alors il existe une isotopie de $\xi_{+}$en une structure $\xi_{+}^{\prime}$ telle que la paire $\left(\xi_{+}^{\prime}, \xi_{-}\right)$soit normale et fortement tendue et que le lieu des contacts $\Delta_{+}$soit transversal à $\xi_{+}^{\prime}$ et $\xi_{-}$(en particulier, il n'y a pas de naissance-mort).

Démonstration C'est le cas relatif de la preuve de la proposition 3.5. On relie les contacts quadratiques par une collection d'arcs positivements transversaux à $\xi_{ \pm}$qui évitent $\Delta_{+}$. Près des contacts quadratiques, on prend soin que ces arcs soient situés dans le demi-espace, délimité par $\xi_{+}=\xi_{-}$, qui ne contient pas $\Delta_{+}$. En particulier, la direction de $X$ le long de ces arcs converge au bord vers la direction de la demi-séparatrice 
de type selle des naissances-morts. On modifie alors $\xi_{+}$et $\xi_{-}$le long de chacun de ces arcs pour faire apparaître deux familles de contacts parallèles, l'une constituée de foyers, l'autre de selles, qui permettent de faire disparaître les naissances-morts.

Remarque 7 Dans le cas général, si $\Delta$ est sans contact quadratique avec $\xi_{ \pm}$, on peut définir sur $V \backslash \Delta$ deux champs de plans transversaux, qui se comportent le long de $\Delta$ à la manière du plan tangent à un feuilletage d'énergie finie près de son lieu singulier [12].

Exemple $\operatorname{Si}(K, \theta)$ est une décomposition de $V$ en livre ouvert, la construction de Thurston et Winkelnkemper [16], systématisée par Giroux dans [8], fournit une paire $\left(\xi_{+}, \xi_{-}\right)$de structures de contact portées par $(K, \theta)$ pour laquelle $\Delta_{-}=K$ (et est constitué de foyers) et dont la restriction à $V \backslash N(K)$ est fortement tendue (voir [8] pour une description de ces notions). À l'aide de la proposition 3.6, on peut également en obtenir une (normale) pour laquelle de surcroît $\Delta_{+}$est transversale à $\xi_{+}$, ainsi qu'aux pages de $(K, \theta)$.

Si $\lambda$ est uniquement intégrable, et si $\left(\xi_{+}, \xi_{-}\right)$est normale et fortement tendue, alors le feuilletage intégral $\mathcal{F}$ de $\lambda$ est tendu. Comme les structures $\xi_{ \pm}^{t}$ convergent vers $\mathcal{F}$, elles sont tendues et aussi $\xi_{+}$et $\xi_{-}$.

Réciproquement, si $\xi_{+}$et $\xi_{-}$sont tendues, forment une paire normale et si $\lambda$ est uniquement intégrable, alors $\lambda$ est sans composante de Reeb homologue à zéro. Dans le cas où la variété $V$ est une sphère d'homologie rationnelle atoroïdale, $\mathcal{F}$ est tendu. On peut alors montrer que la paire $\left(\xi_{+}, \xi_{-}\right)$peut être déformée (par une isotopie de $\xi_{+}$) en une paire fortement tendue.

Proposition 3.7 Soit $\left(\xi_{+}, \xi_{-}\right)$une paire normale positive de structures tendues sur une sphère d'homologie rationnelle atoroïdale. Si $\lambda$ est uniquement intégrable, alors $\left(\xi_{+}, \xi_{-}\right)$est déformable, par une isotopie de $\xi_{+}$, en une paire normale et fortement tendue.

Démonstration Comme on l'a déjà signalé plus haut, sous les hypothèses de la proposition 3.7, le théorème 3.3 implique que le feuilletage intégral de $\lambda$ est sans composante de Reeb. De plus, comme la variété ambiante est atoroïdale, il est sans composante de Reeb généralisée, c'est-à-dire qu'il est tendu.

Il suffit de montrer que pour $t$ assez grand, la paire $\left(\xi_{+}^{t}, \xi_{-}^{t}\right)$ est fortement tendue. Pour cela, on va utiliser un système d'arcs transversal à $\lambda$ et la proximité entre $\lambda$ et $\xi_{ \pm}^{t}$. 
On se donne deux familles de boules $\left(B_{i}(1)\right)_{1 \leq i \leq n}$ et $\left(B_{i}(2)\right)_{1 \leq i \leq n}$ dans $V$ avec les propriétés suivantes:

- $\operatorname{Int}\left(B_{i}(1)\right)$ recouvre $V$;

- $B_{i}(1) \subset \operatorname{Int} B_{i}(2)$;

- il existe un difféomorphisme $\phi: B_{i}(2) \rightarrow B(0,2) \subset \mathbb{R}^{3}$, avec $\phi\left(B_{i}(1)\right)=B(0,1)$ et $\phi_{*} \lambda$ est $\epsilon-C^{0}$-proche de $\{d z=0\}$.

Sous ces hypothèses, si $\epsilon$ est petit, on peut joindre tout point de $B_{i}(1)$ (resp. le pôle sud de $B_{i}(2)$ ) au pôle nord de $B_{i}(2)$ (resp. tout point de $B_{i}(1)$ ) par un arc positivement transversal à $\lambda$, dont l'angle avec $\lambda$ est en tout point supérieur à une certaine constante $c$.

En particulier, si $t$ est assez grand ( $>t_{0}$ ), on peut joindre tout point de $B_{i}(1)$ (resp. le pôle sud de $B_{i}(2)$ ) au pôle nord de $B_{i}(2)$ (resp. tout point de $\left.B_{i}(1)\right)$ par un arc positivement transversal à $\xi_{+}$et $\xi_{-}$.

On se donne alors pour tout couple $(i, j), 1 \leq i, j \leq n$, deux arcs qui relient les pôles nord et sud de $B_{i}(2)$ aux pôles sud et nord de $B_{j}(2)$ et qui sont positivement transversaux à $\lambda$. L'existence de ces arcs est assurée par le fait que le feuilletage intégral de $\lambda$ est tendu. À nouveau, si $t$ est assez grand ( $\left.>t_{1}\right)$, chacun de ces arcs est positivement transversal à $\xi_{+}^{t}$ et $\xi_{-}^{t}$. Pour conclure, on remarque que si $t>\max \left(t_{0}, t_{1}\right)$, et si $x \in B_{i}(1), y \in B_{j}(1)$ sont deux points quelconques de $V$, on peut joindre $x$ à $y$ par un chemin transversal à $\xi_{+}$et $\xi_{-}$en concaténant un chemin entre $x$ et le pôle nord de $B_{i}(2)$, le chemin entre le pôle nord de $B_{i}(2)$ et le pôle sud de $B_{j}(2)$ et un chemin entre le pôle sud de $B_{i}(2)$ et $y$.

Remarque 8 L'isotopie qui déforme $\xi_{+}$ne préserve pas $\xi_{-}$et on ne peut donc pas dire a priori que la paire initiale est fortement tendue.

\section{Variétés à bords sphériques}

Dans le cas des variétés bordées par une sphère, se donner une paire positive tendue "convexe au bord" est très fortement contraignant pour la variété. On notera un analogue frappant de ce résultat obtenu via une méthode de remplissage par des disques holomorphes [4].

Théorème 4.1 Soit $V$ une variété compacte connexe de dimension trois avec $\partial V=S^{2}$. Si on suppose que $V$ porte une paire positive $\left(\xi_{+}, \xi_{-}\right)$de structures tendues telle que $\Delta_{+}$rencontre $\partial V$ transversalement en deux points $S$ et $N$ et que $X$ soit strictement sortant de $V$ le long de $\partial S \backslash(S \cup N)$, alors $V \simeq B^{3}$. 
Démonstration D'après la proposition 2.1, on peut se ramener au cas où la paire $\left(\xi_{+}, \xi_{-}\right)$est normale. La condition au bord nous dit que le champ de plans $\lambda$ est bien défini sur $V$. Il est transversal à $\partial V$ en dehors de $N$ et $S$. La courbe $\Delta_{+}$rencontre $\partial V$ aux points $N$ et $S$ en un foyer de $X$. En particulier, $\lambda$ est uniquement intégrable, lisse, et s'intègre en un feuilletage en disques au voisinage de ces pôles.

Soit $A$ l'ensemble des points de $V$ qui appartiennent à un disque intégral de $\lambda$, de classe nulle dans $H_{2}(V, \partial V, \mathbb{Z})$ et le long duquel $\lambda$ est uniquement intégrable. On montre que $A$ est ouvert et fermé dans $V$, et donc égal à $V$ par connexité de $V$.

Lemme 4.2 $A$ est fermé.

Démonstration Soit $x$ un point de $V$, limite d'une suite $\left(x_{n}\right)_{n \in \mathbb{N}}$ de points de $A$, situés tous "au-dessus" de $x$, par rapport à $\lambda(x)$, c'est-à-dire que, si $D_{n}$ désigne le disque intégral de $\lambda$ passant par $x_{n}, x$ est situé dans la composante de $V \backslash D_{n}$ qui contient $S$. On note $D$ l'ensemble des points d'adhérence de la famille $D_{n}$.

Si $y \in D$ est la limite d'une suite $\left(y_{\phi(n)}\right)$ de points de $D_{\phi(n)}$, alors dans un petit voisinage cylindrique $U=D^{2} \times[-1,1]$ de $y=(0,0,0)$, la composante de $D_{\phi(n)} \cap U$ qui contient $y_{\phi(n)}$ est le graphe d'une fonction $f_{\phi(n)}$ de classe $C^{1}$ au-dessus de $D^{2}$, et qui est strictement positive en $(0,0)$ car $x$ est au-dessous des disques $D_{n}$, et donc aussi $y$. D'après le théorème d'Ascoli, une sous-suite de la suite $\left(f_{\phi(n)}\right)$ converge uniformément vers une fonction $f$. C'est alors aussi le cas de la suite elle-même car les graphes sont deux à deux disjoints. La suite des plans tangents aux graphes des $f_{\phi(n)}$ converge elle aussi, car c'est $\left.\lambda\right|_{D_{\phi(n)}}$, et on en déduit que le graphe de $f$ est un disque intégral de $\lambda$, inclus dans $D$. On remarque maintenant que la composante connexe de $D \cap U$ qui contient $y$ est égale au graphe de $f$. Ceci résulte du fait que les disques $D_{n}$ sont deux à deux disjoints, car $\lambda$ est uniquement intégrable le long de $D_{n}$, et tous situés au-dessus du graphe de $f$.

En conclusion, on obtient que $D$ est une surface intégrale de $\lambda$. Un argument de Haefliger [10] fournit que $D$ est compacte, car limite de feuilles compactes. Comme elle est limite de disques, on obtient alors également que $D$ est un disque de classe $C^{1}$. Son feuilletage caractéristique $\lambda D=\xi_{+} D=\xi_{-} D$ dirigé par $X$ est sortant le long du bord. Comme $\xi_{ \pm}$sont tendues, il ne possède pas de cycle limite et toutes les feuilles sont issues d'une singularité de $\xi_{ \pm} D=\Delta_{+} \cap D$. C'est même le cas de toute orbite passant au voisinage de $D$, comme dans le lemme 4.3. Le plan $\lambda$ est donc uniquement intégrable le long de $D$ d'après le théorème 3.4. On raisonne de même lorsque $x$ est situé "au-dessus" des disques de la famille $\left(D_{n}\right)$, ce qui permet de conclure dans tous les cas.

Algebraic \& $\mathcal{G}$ eometric Topology, Volume 11 (2011) 
Lemme 4.3 A est ouvert.

Démonstration Si $x \in A$ est situé sur un disque intégral $D$ de $\lambda$, toutes les feuilles de $\xi_{ \pm} D$ proviennent d'une singularité de $\xi_{ \pm} D$, c'est-à-dire d'un point de $D \cap \Delta_{+}$ (comme $\xi_{ \pm}$sont tendues, $\xi_{ \pm} D$ ne contient pas de cycle limite et par ailleurs $\xi_{ \pm} D$ est transversal à $\partial D)$.

Si $\xi_{ \pm} D$ est de type Morse-Smale, toutes les orbites passant par un point proche de $D$ sont issues de $\Delta_{+}$(et même de foyers ou de selles de $\Delta_{+}$). Ceci implique, via le théorème 3.4 , que $\lambda$ est uniquement intégrable dans un voisinage de $D$. Un voisinage de $D$ est donc feuilleté par des disques intégraux de $\lambda$ d'après le théorème de stabilité de Reeb.

Si $\xi_{ \pm} D$ possède une naissance-mort $n$, elle est génériquement unique sur $D$. Celle-ci est située sur une branche $\Delta$ de $\Delta_{+}$. La séparatrice stable de $n$ provient d'un foyer $e$ de $\xi_{ \pm} D$ situé sur une composante $\Delta^{\prime}$ de $\Delta_{+}$. D'un côté de $D$, les orbites proches de celles qui proviennent de $n$ sont toutes issues d'un voisinage de $n$ dans $\Delta$, et de l'autre côté de $D$ elles sont toutes issues d'un voisinage de $e$ dans $\Delta^{\prime}$. Comme précédemment, on conclut en remarquant que $X$ est transitif au voisinage de $D$.

La même propriété de transitivité au voisinage de $D$ est vérifiée si $\xi_{ \pm} D$ présente une connexion entre deux selles.

Ceci montre que $V$ est feuilletée par des disques et termine la preuve du théorème 4.1.

Problème Peut-on montrer que si $V$ porte une paire positive de structures de contact tendues, alors elle est irréductible?

\section{Bibliographie}

[1] D Bennequin, Entrelacements et équations de Pfaff, from: "Third Schnepfenried geometry conference, Vol. 1 (Schnepfenried, 1982)”, Astérisque 107, Soc. Math. France (1983) 87-161 MR753131

[2] V Colin, Structures de contact tendues sur les variétés torö̈dales et approximation de feuilletages sans composante de Reeb, Topology 41 (2002) 1017-1029 MR1923997

[3] Y Eliashberg, Contact 3-manifolds twenty years since J Martinet's work, Ann. Inst. Fourier (Grenoble) 42 (1992) 165-192 MR1162559

[4] Y Eliashberg, H Hofer, A Hamiltonian characterization of the three-ball, Differential Integral Equations 7 (1994) 1303-1324 MR1269658

[5] Y Eliashberg, W P Thurston, Confoliations, Univ. Lecture Series 13, Amer. Math. Soc. (1998) MR1483314

Algebraic 83 Geometric Topology, Volume 11 (2011) 
[6] E Giroux, Convexité en topologie de contact, Comment. Math. Helv. 66 (1991) 637-677 MR1129802

[7] E Giroux, Structures de contact en dimension trois et bifurcations des feuilletages de surfaces, Invent. Math. 141 (2000) 615-689 MR1779622

[8] E Giroux, Géométrie de contact: de la dimension trois vers les dimensions supérieures, from: "Proceedings of the International Congress of Mathematicians, Vol. II (Beijing, 2002)", (T Li, editor), Higher Ed. Press, Beijing (2002) 405-414 MR1957051

[9] J W Gray, Some global properties of contact structures, Ann. of Math. (2) 69 (1959) 421-450 MR0112161

[10] A Haefliger, Variétés feuilletées, Ann. Scuola Norm. Sup. Pisa (3) 16 (1962) 367-397 MR0189060

[11] P Hartman, Ordinary differential equations, Wiley, New York (1964) MR0171038

[12] H Hofer, K Wysocki, E Zehnder, Finite energy foliations of tight three-spheres and Hamiltonian dynamics, Ann. of Math. (2) 157 (2003) 125-255 MR1954266

[13] Y Mitsumatsu, Anosov flows and non-Stein symplectic manifolds, Ann. Inst. Fourier (Grenoble) 45 (1995) 1407-1421 MR1370752

[14] Y Mitsumatsu, Foliations and contact structures on 3-manifolds, from: "Foliations: geometry and dynamics (Warsaw, 2000)", (P Walczak, L Conlon, R Langevin, T Tsuboi, editors), World Sci. Publ., River Edge, NJ (2002) 75-125 MR1882766

[15] G Perelman, The entropy formula for the Ricci flow and its geometric applications arXiv:math/0211159

[16] W P Thurston, HE Winkelnkemper, On the existence of contact forms, Proc. Amer. Math. Soc. 52 (1975) 345-347 MR0375366

[17] S Zannad, Surfaces branchées en géométrie de contact, $\mathrm{PhD}$ thesis, Université de Nantes (2006) Available at http://tel.archives-ouvertes.fr/tel-00103561/

[18] S Zannad, A sufficient condition for a branched surface to fully carry a lamination, Algebr. Geom. Topol. 7 (2007) 1599-1632 MR2366173

Laboratoire de Mathématiques Jean Leray, UMR 6629 du CNRS, Université de Nantes

2 rue de la Houssinière, F-44322 Nantes, France

Instituto de Matemática, Universidade Federal Fluminense

Rua Mário Santos Braga, Valonguinho 24.020-140, Niterói RJ, Brazil

vincent.colin@univ-nantes.fr, firmo@mat.uff.br

http://www.math.sciences. univ-nantes.fr/ vcolin/

Received: 2 June 2009

Algebraic $8 \mathcal{G}$ Geometric Topology, Volume 11 (2011) 